\title{
Concentración en el Gran Santiago y migración: población, vivienda y recursos humanos 1977-2017
}

\author{
Jorge Rodríguez-Vignoli. Centro Latinoamericano y Caribeño de Demografía \\ (Celade-División de Población de la Cepal), Santiago, Chile.
}

RESUMEN | Santiago concentra una fracción significativa de los recursos económicos y humanos de Chile, pero hay debate sobre la persistencia de esta concentración. La migración es decisiva para la redistribución y desconcentración de los recursos humanos. Para examinar empíricamente la eventual desconcentración demográfica de Santiago, precisar sus alcances territoriales y estimar el papel de la migración en ella, se explotan los microdatos de los últimos cuatro censos de población y vivienda oficiales del país, con los cuales se generan indicadores clave. Además, se analizan otras dimensiones de la desconcentración, en particular la habitacional. Los resultados sugieren que la migración interna efectivamente abona a un proceso de desconcentración real de Santiago desde fines del siglo pasado, aunque esto ha sido contrarrestado recientemente por la inmigración internacional. Adicionalmente, la inflexión hacia la pérdida migratoria de Santiago ha sido segmentada y, paradójicamente, ha contribuido a reforzar la base de recursos humanos jóvenes y calificados de la ciudad.

PALABRAS CLAVE | concentración espacial, migración, metropolización.

ABSTRACT $\mid$ A high percentage of Chilean economic and human resources are concentrated in Santiago, but there is debate about this pattern's persistence. Migration is a crucial driver of the redistribution and deconcentration of the spatial distribution of the population. To empirically examine Santiago's potential deconcentration, specify its territorial scope, and estimate the role of migration in this process, microdata of the last four official censuses of the country are exploited to produce traditional and innovative indicators. Also, other dimensions of deconcentration are analyzed, particularly housing. The results suggest that internal migration has supported Santiago's real deconcentration since the end of the last century. However, this effect has been counteracted by international in-migration. Additionally, the swing towards net out-migration from Santiago has differed among population groups and, paradoxically, has contributed to strengthening the base of young and skilled human resources in the city.

KEYWORDS | spatial concentration, migration, metropolization. 


\section{Introducción, antecedentes y preguntas guía}

Las ciudades grandes de América Latina experimentaron profundas mutaciones en las últimas décadas del siglo pasado (Aguilar, 2006; Aguilar \& Escanilla, 2011; Cepal, 2012; Cunha, 2018; Cunha \& Rodríguez, 2009; De Mattos, 2010; Dureau et al., 2002; Dureau et al., 2014; Ribeiro, 2015; Roberts \& Wilson, 2009; Rodríguez-Vignoli, 2019b; Villa \& Rodríguez-Vignoli, 1997). Desde el fin de la Segunda Guerra Mundial hasta la década de 1980, las ciudades grandes lideraron la expansión económica, ampliaron y renovaron sus capacidades productivas, registraron significativos avances sociales y urbanos, concentraron la apertura de servicios clave -como educación, salud, tecnología e innovación-y se convirtieron en imanes para los migrantes. Sin embargo, esta combinación aparentemente virtuosa tenía debilidades estructurales en todos los frentes, siendo particularmente notorias la relativa a la absorción laboral y residencial de los inmigrantes, así como la referida a la gestión y planificación de las ciudades. En el caso de los inmigrantes, su vida y la de su descendencia tendía a mejorar y, sobre todo, a tener, en promedio, mayores y mejores oportunidades que en sus lugares de origen. Sin embargo -además de los casos diferentes al promedio, es decir, de quienes empeoraban su situación-, en general muchos inmigrantes a las grandes ciudades experimentaban dificultades para acceder a puestos de trabajo formales y para ejercer sus derechos básicos, incluyendo el de vivienda, así como el más amplio derecho a la ciudad. Por su parte, las capacidades financieras, técnicas e institucionales para planificar, gestionar y administrar las grandes ciudades fueron insuficientes para enfrentar su crecimiento explosivo y empujado por una inmigración de ciudadanos muchas veces expulsados de sus lugares de origen y con escasa preparación para enfrentar los desafíos de su nuevo hábitat. Por otra parte, la gestión metropolitana fue infructuosa, en muchos casos miope o hasta ciega, en sus objetivos de: i) intervenir la ciudad y modificar su estructura y hacerla más apta para absorber este crecimiento explosivo, y ii) superar, o al menos atenuar, las profundas limitaciones en materia de infraestructura, equipamiento, normatividad, servicios y funcionamiento en general de la ciudad. Ciertamente, las desigualdades históricas de la región se expresaron en las grandes ciudades en términos de disparidades intrametropolitanas, en sus dimensiones económicas, institucionales, infraestructurales y políticas, desigualdades que muchas veces fueron reforzadas por la acción pública y la inversión privada.

Todo lo anterior devino crítico en la década de 1980, la denominada "década perdida” para América Latina, cuando una profunda recesión gatilló recortes de gasto e inversión pública y privada, quiebra de industrias y debilitamiento de la gestión y la planificación metropolitana, con incrementos severos de la pobreza y el desempleo y, al mismo tiempo, se agudizaron diversos problemas metropolitanos (congestión, contaminación, criminalidad, inseguridad, hacinamiento, segregación, etc.), enmarcados en las debilidades estructurales acumuladas (exclusión, desigualdad, marginalidad, informalidad, corrupción). Esta tormenta perfecta erosionó el atractivo migratorio de las ciudades grandes y llevó a pronósticos fatalistas de ciudades apocalípticas, por la combinación de envergaduras enormes y la multiplicación de problemas aparentemente inmanejables. 
Contra estos pronósticos, desde fines de la década de 1990 y durante las dos primeras décadas del siglo XXI se han verificado procesos de recuperación metropolitana que, al menos, han permitido contener su caída libre. Por cierto, lo anterior en modo alguno significa que los problemas hayan desaparecido; de hecho, la mayoría ha permanecido y algunos incluso han aumentado. Tampoco implica que el atractivo migratorio haya repuntado; en efecto, siguió cayendo según las cifras comparativas disponibles (Chávez et al., 2016; Rodríguez-Vignoli, 2017, 2019b; base de datos MIALC). Pero el escenario actual ya no es tan pesimista como el que prevalecía a finales del siglo pasado, y a ello se han sumado visiones renovadas desde la década de 1990, que subrayan el potencial de estas ciudades en la nueva economía global y de servicios (De Mattos, 2010; Florida, 2005; Pacione, 2009; Sassen, 1991, 2007).

Santiago ha sido una ciudad particularmente expuesta a este ciclo de expansión-crisis-renovación, habida cuenta su ingreso temprano al nuevo escenario económico mundial, marcado por el capitalismo global, la "financiarización" y la revolución tecnológica. En este sentido, la ciudad sufrió embates durísimos producto de la reestructuración económica del país iniciada durante dictadura desde 1973. La decadencia de la industria nacional ante la competencia extranjera y el debilitamiento del sector público se sintieron con particular fuerza en la capital del país. La crisis económica de 1981-1984 causó un salto del desempleo y probablemente de la pobreza, así como estragos en la inversión pública. Todo lo anterior influyó en que problemas acumulados previamente, pero desatendidos por la falta de inversión y de políticas específicas, como la contaminación y la congestión, aumentaran explosivamente durante las décadas de 1980 y 1990. Asimismo, la política habitacional, si bien fue efectiva en materia de construcción de viviendas sociales (aunque con estándares de calidad más bien insuficientes), tuvo, en general, deficiencias en términos de construcción de ciudad y de derecho a la ciudad, e incentivó una rápida y escasamente planificada expansión territorial de la ciudad, un alejamiento de la población beneficiada con el subsidio habitacional de sus lugares de trabajo y un aumento de los tiempos de transporte y la congestión (Dureau et al., 2002; Dureau et al., 2014; Fuentes et al., 2017; Galetovic \& Jordán, 2006; Rodríguez-Vignoli \& Espinoza, 2012; Rodríguez-Vignoli \& Rowe, 2018b; Rodríguez \& Sugranyes, 2005; Villa \& Rodríguez-Vignoli, 1997).

Con todo, el crecimiento económico del país desde fines de la década de 1980, así como políticas públicas activas y efectivas para reducir la pobreza y enfrentar otros problemas sociales y urbanos, han dado nuevos bríos a la ciudad. De hecho, Santiago se posiciona actualmente con varios indicadores positivos en el contexto latinoamericano. Además, sobresale en América Latina en casi todos los índices de condiciones de vida, formalidad, competitividad y dinamismo económico y tecnológico (De Mattos, 2010; Fuentes et al., 2017; Galetovic \& Jordán, 2006). Con todo, estos indicadores e índices invisibilizan la enorme desigualdad en su interior, así como la persistencia de problemas y déficits de distinto tipo que complican el funcionamiento de la ciudad y afligen a sus moradores (De Mattos \& Hidalgo, 2007; Rodríguez-Vignoli \& Espinoza, 2012; Rodríguez-Vignoli \& Rowe, 2018b; Rodríguez-Vignoli et al., 2017). 
Este conjunto de tendencias complejas, contrapuestas en algunos casos, sumado a los debates sobre las fuerzas y procesos estructurales de desconcentración económica territorial del capitalismo global (Atienza \& Aroca, 2012; Cunha, 2018; Frey, 1987; Fujita \& Krugman, 2004; Fujita, Krugman \& Venables, 2000; Harvey, 2014; Ingram, 1998; Ribeiro, 2015; Rodríguez-Vignoli, 2017; Sassen, 1991; Williamson, 1965), alimenta dudas sobre la persistencia de la concentración de la economía y la población en Santiago, así como sobre su atractivo migratorio reciente.

Los datos del Censo 2017 y su comparación con los tres censos oficiales previos permiten una descripción actualizada y relativamente detallada de las tendencias de la concentración demográfica en Santiago y su atractivo migratorio. En esa línea, a continuación se hará una análisis sistemático de esta fuente para responder las siguientes preguntas: i) cómo ha evolucionado la concentración demográfica en Santiago, qué papel ha desempeñado la migración en ella y qué relación ha tenido la concentración demográfica en Santiago con la concentración habitacional allí; ii) cuál es el alcance territorial de los intercambios migratorios de Santiago "cercano" o "lejano" y cómo estos se relacionan con una potencial "desconcentración concentrada” (Cunha y Rodríguez, 2009); iii) cómo ha evolucionado el efecto de la migración sobre los recursos humanos de Santiago, en particular su composición etaria y educativa, ya que podría estar aconteciendo una desconcentración selectiva -por ejemplo de toda la población en promedio, pero no de ciertos grupos, como los de mayor instrucción o recursos-, en cuyo caso el análisis e interpretación de tal desconcentración debiera considerar esta distinción.

\section{Marco metodológico}

La principal fuente de datos del estudio corresponde a las bases de microdatos de los cuatro últimos censos oficiales con que cuenta el país, levantados en abril de 1982, 1992, 2002 y 2017. Los cuatro fueron de hecho y se levantaron en un día (hubo un plazo algo mayor para las zonas rurales). En todos estos censos se capta la migración con la pregunta por lugar de residencia cinco años antes del censo (medición de "fecha fija" en la jerga demográfica) y con una desagregación máxima hasta la escala de comuna. Se usó esta fuente y esta pregunta porque son las únicas que permiten estimar las tasas y los efectos de la migración a escala de comuna (Bilsborrow, 2016; Celade-prolap, 1998; Rees et al., 2000; Rodríguez-Vignoli, 2009; Rodríguez-Vignoli \& Rowe, 2018a; Villa, 1991), que es lo que se requiere para este estudio, que "construye" varias definiciones territoriales de Santiago en la base de datos censal, a partir de las comunas que las componen.

Para procesar los microdatos censales, se utilizó el paquete computacional libre Redatam versión 7.0, desarrollado y mantenido por CELADE-División de Población de la CEPAL (www.cepal.org/es/temas/redatam). La primera tarea fue generar las variables de residencia habitual y residencia cinco ańos antes del censo a escala de comuna. Se crearon numerosas variables de residencia habitual y residencia anterior, en función de las agregaciones territoriales necesarias para construir las diferentes modalidades de matrices de migración requeridas para responder empíricamente a las preguntas antes planteadas. Del cruce de esas variables, y en algunos casos cruces 
con otras variables o ponderación con otras variables, surgen las matrices usadas para estimar flujos, saldos migratorios y efectos de la migración.

Una vez generadas y limpiadas de códigos inválidos o no aplicables y de inmigrantes internacionales (en rigor, de quienes residían en otro país cinco años antes del censo), las nuevas variables de comuna de residencia habitual al momento del censo y comuna de residencia cinco años antes, se usaron para construir matrices de migración. Se trata de varias matrices de origen-destino dependiendo del grado de desagregación usado y también del objetivo del análisis. En el caso más frecuente, el de mayor agregación, la matriz es de 2 × 2 con Santiago -más concretamente una o más de sus definiciones territoriales- como una entidad, y el resto de los municipios del país (incluyendo los de la Región Metropolitana que no forman parte de Santiago) agrupados como la otra entidad. En otros casos las matrices tienen dimensiones mayores, por ejemplo, cuando se examina la migración entre Santiago y ciudades cercanas y lejanas a ella para aproximarse a una evaluación empírica de la hipótesis de la desconcentración concentrada.

Respecto de las definiciones territoriales, el Área Metropolitana del Gran Santiago (AMGS) corresponde a las 34 comunas de la Región Metropolitana que desde hace bastante tiempo se consideran conurbadas (Rodríguez-Vignoli \& Rowe, 2018b). El Área Metropolitana del Gran Santiago Extendida (AMGS-E), que es el foco de este estudio, por su parte, corresponde a 49 comunas de las 52 que componen la Región Metropolitana actualmente. Los antecedentes y justificaciones de esta definición se encuentran en De Mattos, Fuentes y Link, 2016; Galetovic y Jordán, 2006; Rodríguez-Vignoli y Espinoza, 2012; Rodríguez-Vignoli y Rowe, 2018b; entre otros. En la Figura 1 se presenta la definición del AMGs-E bajo la modalidad de grandes zonas para facilitar el contraste entre el Amgs y el Amgs-E. Las zonas 1 a 5 (Centro, Pericentro, Periferia tradicional, Barrio Alto y Periferia elitizada) conforman el Amgs; y las zonas 1 a 11 (se suman los cinco suburbios y Melipilla) conforman el AMGs-E. Finalmente, hay una tercera definición que se denomina Santiago-MIALC, que se usará para examinar la hipótesis de la "desconcentración concentrada" mediante las matrices de migración entre ciudades de la base de datos MIALC de CELADE (https:// celade.cepal.org/bdcelade/mialc/), que está compuesta por las 34 comunas del AMGs más 10 comunas adicionales de otras provincias de la Región Metropolitana, que se consideran aglomeradas en la última versión de dicha base de datos.

Se decidió trabajar con varias definiciones territoriales de la ciudad para garantizar que los resultados obtenidos no dependan de la delimitación de la ciudad. Esto último suele ser un asunto contencioso, por una parte, y decisivo para los resultados, por otra. Cabe destacar que, en esta investigación, la definición territorial opera a escala de comunas por una razón metodológica ya insinuada: la migración es la variable de interés del estudio y en los censos de Chile (como en la mayoría de los censos de América Latina), la mayor desagregación territorial para la cual se capta la migración es la escala de comuna. 
FIGURA I | AMGS-E, "grandes zonas": comunas componentes

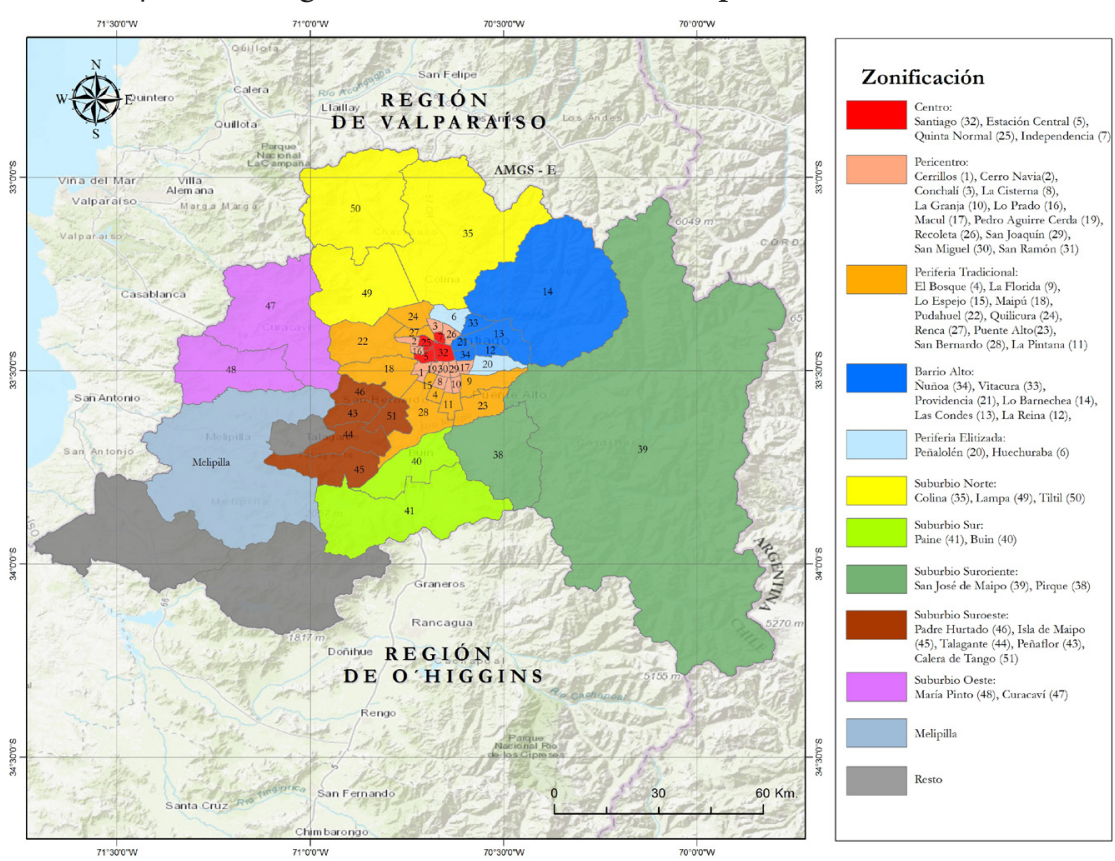

FUENTE: RODRÍGUEZ-VIGNOLI Y ROWE (20I8B, P. 52)

Entonces, con las matrices antes descritas e indicadores de crecimiento demográfico y cambio socioeconómico complementarios, se estiman, en primer lugar, los flujos migratorios, la inmigración, la emigración y el saldo migratorio y las respectivas tasas de migración para el AMGs y el AMGs-E, en los periodos 1977-1982, 19871992, 1997-2002 y 2012-2017. Estos resultados permiten, en primer lugar, evaluar las tendencias del atractivo migratorio de Santiago y su relación con la hipótesis de la desconcentración del sistema de asentamiento humanos en Chile. Estas tendencias se evalúan según edad y nivel educativo como primera aproximación a la potencial desconcentración de los recursos humanos, y no solo la población. Luego, se evalúa la hipótesis de la desconcentración concentrada, usando las matrices de migración entre ciudades de MIALC, es decir, considerando la definición de 44 comunas de Amgs-mialc. Posteriormente, se examina la evolución de la concentración del parque habitacional para establecer nexos entre las tendencias demográficas y migratorias y las habitacionales. La parte empírica del estudio concluye con la aplicación del procedimiento elaborado por Rodríguez-Vignoli (2013) y ampliado, refinado y formalizado recientemente por Rodríguez-Vignoli y Rowe (2018a) para estimar el efecto neto y exclusivo de la migración (neta, inmigración y emigración) sobre la composición etaria y educativa del AMGs-E en los periodos 1977-1982, 1987-1992, 1997-2002 y 2012-2017, que permite un veredicto más preciso sobre la eventual desconcentración de los recursos humanos, sobre todo los calificados. Para más detalles sobre el procedimiento y sus supuestos, interpretación, fortalezas y debilidades, véase Rodríguez-Vignoli y Rowe (2018a, 2018b). 


\section{Concentración demográfica y habitacional y atractivo migratorio según edad y educación}

El indicador más intuitivo para evaluar la tendencia de la concentración demográfica en el AMGS-E es el porcentaje que su población representa en el total nacional. De acuerdo con la Figura 2, este porcentaje ha tenido un aumento sostenido durante todo el periodo de observación, precedido de una tendencia similar durante todo el siglo xx (Galetovic \& Jordán, 2006; Rodríguez-Vignoli \& Rowe, 2018b; Villa \& Rodríguez-Vignoli, 1997; base de datos Depualc: https://celade.cepal.org/bdcelade/depualc/), que alcanzó un 40,2\% en 2017 . Este porcentaje puede variar dependiendo de la definición usada. Por ejemplo, de acuerdo con la definición vigente de la base de datos que coincide con la definición de Santiago-MIALC, ese porcentaje sería de 38\% en 2017. De cualquier manera, usando esa definición, la tendencia de la concentración demográfica en Santiago es la misma: aumento sistemático desde el Censo 1952. Por ende, la primera respuesta a la hipótesis de la desconcentración demográfica es que no ha habido tal, considerando esta definición actualizada y extendida de la ciudad.

FIGURA 2 | AMGS-E: porcentaje sobre la población nacional y urbana, 1982, 1992,2002 y 2017

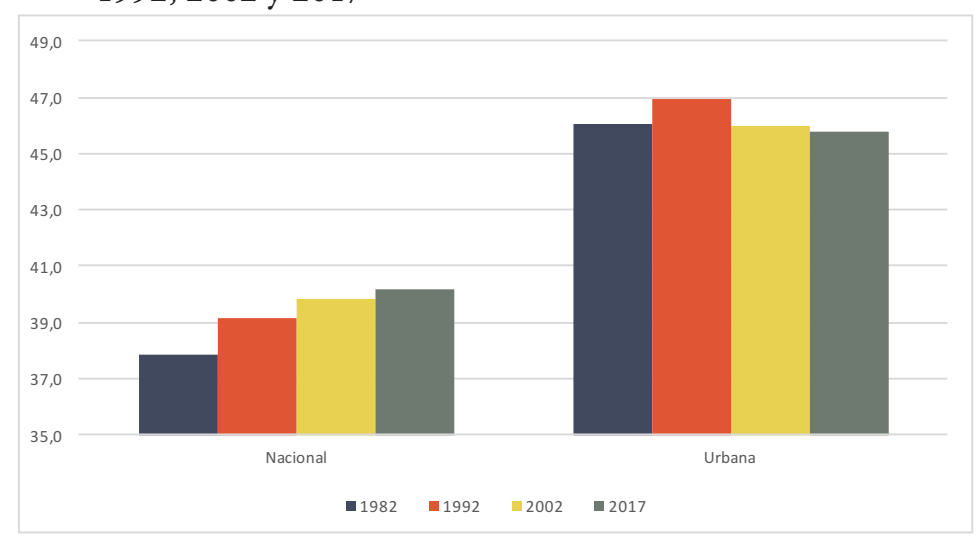

NOTA: POBLACIÓN CENSADA, QUE PUEDE DIFERIR DE LA RESIDENTE, PORQUE LOS CENSOS DE CHILE INCLUIDOS EN LA FIGURA FUERON TODOS DE HECHO. NO SE CALCULA SOBRE LA BASE DE RESIDENTES, PORQUE UNA FRACCIÓN DE LA POBLACIÓN (PEQUEÑA) NO RESPONDE ESA PREGUNTA Y PORQUE LOS NIVELES Y TENDENCIAS NO CAMBIAN AL USAR LA VARIABLE DE RESIDENCIA HABITUAL.

FUENTE: CÁlCULOS PROPIOS. PROCESAMIENTO ESPECIAL DE MICRODATOS CENSALES

Sin embargo, un segundo indicador, algo más exigente, sugiere una tendencia diferente. Se trata del porcentaje que representa la población del AMGs-E sobre la población urbana del país. Según la misma Figura 2, este porcentaje tendió a aumentar hasta alcanzar una cúspide en 1992 (casi 47\%), tras lo cual comenzó a decaer hasta llegar a un 45,8\% en 2017. Tras esta evolución hay tasas de crecimiento demográfico del AMGs-E inferiores a las del total urbano, lo que puede deberse a la migración, al crecimiento natural, o a ambos. Dado que en general la transición demográfica 
ha sido bastante transversal en el territorio chileno y entre las ciudades del país, es probable que la migración sea el factor decisivo tras esta inflexión del peso relativo del AMGs-E en la población urbana, por lo cual cabe examinarla con mayor detalle.

La Tabla 1 permite concluir que el AMGs-E ha tenido una gradual pérdida de atractivo migratorio, con una inflexión a fines del siglo pasado, detectada por el Censo 2002, cuando pasó a terreno negativo y una intensificación de la expulsión (solo migrantes dentro de Chile, hay que insistir) en el periodo más reciente (2012-2017).

La Tabla 1 agrega valor a los análisis previos por tres vías.

La primera surge del uso de la definición tradicional (AMGs) y la extendida (actual) de la ciudad (AMGS-E), que permite controlar la eventual distorsión de los resultados a causa del uso de definiciones diferentes. De hecho, ambas definiciones dan la misma respuesta a la pregunta: efectivamente la ciudad pasó a terreno negativo, pues desde fines del siglo pasado (1997-2002) es expulsora de población. Ahora bien, la magnitud de la expulsión, tanto en cuantía como en tasa, varía según la definición, ya que el AMGs tiene un saldo negativo más cuantioso y una tasa negativa más elevada, ${ }^{1}$ lo que refleja que parte de su pérdida migratoria se dirige hacia las comunas añadidas en la definición de 49 comunas del AMGs-E.

Una inspección más detallada de la Tabla 1, enfocada en la tendencia de la migración neta en las dos definiciones, muestra la inversión de la relación migratoria entre el AMGs y los suburbios. Mientras en el periodo 1977-1982 la tasa de migración neta del AMGs fue más alta que la del AMGS-E (reflejando un atractivo menor de los suburbios respecto del AMGs), en el periodo 1987-1992 ocurre lo contrario y en los dos periodos que siguen, cuando se da la inflexión migratoria hacia la expulsión en ambas definiciones territoriales de la ciudad, el AMGS registra una emigración neta mayor. Esto último revela que el AMGs es más expulsora que los suburbios que se suman al AMGs-E, lo que se debe a que una fracción significativa de los emigrantes del AMGs se dirige, justamente, a los suburbios incorporados al AMGS-E, fenómeno esperable de acuerdo con todas las teorías de la evolución las ciudades (Fujita, Krugman \& Venables, 2000; Ingram, 1998; Pacione, 2009). Lo que es más bien excepcional en el caso del AMGs-E, es que este crecimiento centrífugo haya sido concomitante durante buen parte del siglo xxi con un crecimiento centrípeto por recuperación del atractivo migratorio de zonas centrales y su consiguiente redensificación (Rodríguez-Vignoli \& Rowe, 2018b).

El segundo valor agregado de la tabla proviene de la consideración de la inmigración y la emigración como componentes de la migración neta. Y en este sentido Santiago se distingue de otras ciudades de América Latina, donde la pérdida de atractivo migratorio se ha debido exclusivamente a la baja de la inmigración (Rodríguez-Vignoli, 2019b). En efecto, el AMGs-E ha reducido su inmigración fuertemente desde tasas del orden de 13,2 por mil en 1977-1982 a 7,6 en 2012-2017, mientras que la emigración saltó desde 6,5 por mil en 19771982 a 9,9 en 2012-2017. Estos cambios son aún más pronunciados en el AMGs, sobre todo en el caso de la emigración, cuya tasa casi se duplica en el periodo de observación (Tabla 1).

$1 \longdiv { \text { Dado que en el texto se usan valores negativos, cada vez que se aluda al nivel y la tendencia de los } }$ mismos se considerará su valor absoluto. Así, un aumento de la expulsión significará un aumento del valor absoluto de la tasa de emigración neta (contrario al valor matemático de ese cambio, que es de reducción y no aumento). 
Y el tercer valor agregado deriva de la segmentación por edad. Sus resultados son plenamente compatibles con hallazgos recientes de otras ciudades de la región (Rodríguez-Vignoli, 2017, 2019b), que muestran un pertinaz, aunque declinante, atractivo de las grandes ciudades para los jóvenes (15-29 años), que contrasta abiertamente con los otros grupos de edad, que tienden a registrar saldos y tasas mucho menores y, en numerosos casos, negativas en los censos de las décadas de 2000 y de 2010. Exactamente la situación que se aprecia en Santiago, donde ambas definiciones son atractivas para los jóvenes durante todo el periodo de observación y, como contrapartida, ambas son expulsoras de los otros cuatro grupos de edad desde el Censo 1992 (salvo grupo 30-44 en el Censo 1992). Por otra parte, el examen por edad muestra que no hay excepciones etarias al patrón de reducción de la tasa de inmigración y aumento de la tasa de emigración, aunque sí se aprecian algunas especificidades, como la fuerte alza de la emigración de niños y adultos en el AMGS, sugiriendo una creciente selectividad etaria de la misma. Siguiendo las relaciones entre las intensidades migratorias de distintos grupos de edad de las tablas modelo de migración (Rogers \& Castro, 1982), es altamente probable que esta concomitancia responda a la emigración de familias jóvenes con hijos pequeños desde el AMGS a los suburbios del AMGS-E y hacia el resto del país.

Se consolida, así, un hecho estilizado, cual es el atractivo que siguen revistiendo las grandes ciudades para los y las jóvenes de América Latina (Rodríguez-Vignoli, 2017, 2019b). Aunque hay teoría al respecto (Florida, 2005; Rodríguez-Vignoli, 2019b; White, 2016; Williamson, 1988), sus determinantes concretos en el caso de Santiago aún no han sido suficientemente estudiados. Considerando las características de este grupo de edad, es altamente probable que su persistente inmigración neta se asocie, además, a especificidades de su perfil socioeconómico y habitacional y a patrones de localización en la ciudad. Los tres temas podrían ser abordados con detalle en sendas investigaciones futuras, por lo cual en este texto solo se indagarán empíricamente de forma introductoria y genérica en el análisis de vivienda que sigue en este capítulo, y en el siguiente se lo hará a escala desagregada dentro de la ciudad.

Por otra parte, varios autores han subrayado que la migración hacia las grandes ciudades no solo es selectiva en términos etarios, sino también en términos socioeconómicos, en particular educativos (Atienza \& Aroca, 2012; Florida, 2005; Rodríguez-Vignoli \& Rowe, 2018a; White, 2016). En este caso, la hipótesis predominante en los países en desarrollo es la de una selectividad ${ }^{2}$ adversa para las grandes ciudades, a causa del bajo nivel educativo de sus inmigrantes, que normalmente provienen de zonas más pobres y con menor acceso a la educación. Pero tal hipótesis puede resultar desactualizada, por basarse en la experiencia del flujo masivo de población del campo a las grandes ciudades, que desde fines del siglo pasado perdió vigencia en América Latina, y más aún en Santiago. En ese sentido, la Tabla 2 presenta una síntesis de las cuantías y tasas de la migración neta, inmigración y emigración, similar a la Tabla 1, pero ahora segmentadas por nivel educativo. ${ }^{3}$

2 O “diferencialidad” en términos estrictos (Rodríguez-Vignoli, 2019b, p. 43).

3 Años de escolaridad recodificados en cuatro categorías desde baja escolaridad ( 0 a 4 años de estudio) a alta escolaridad (13 y más ańos de estudio) para la población de 25 años y más (véase Tabla 2). 


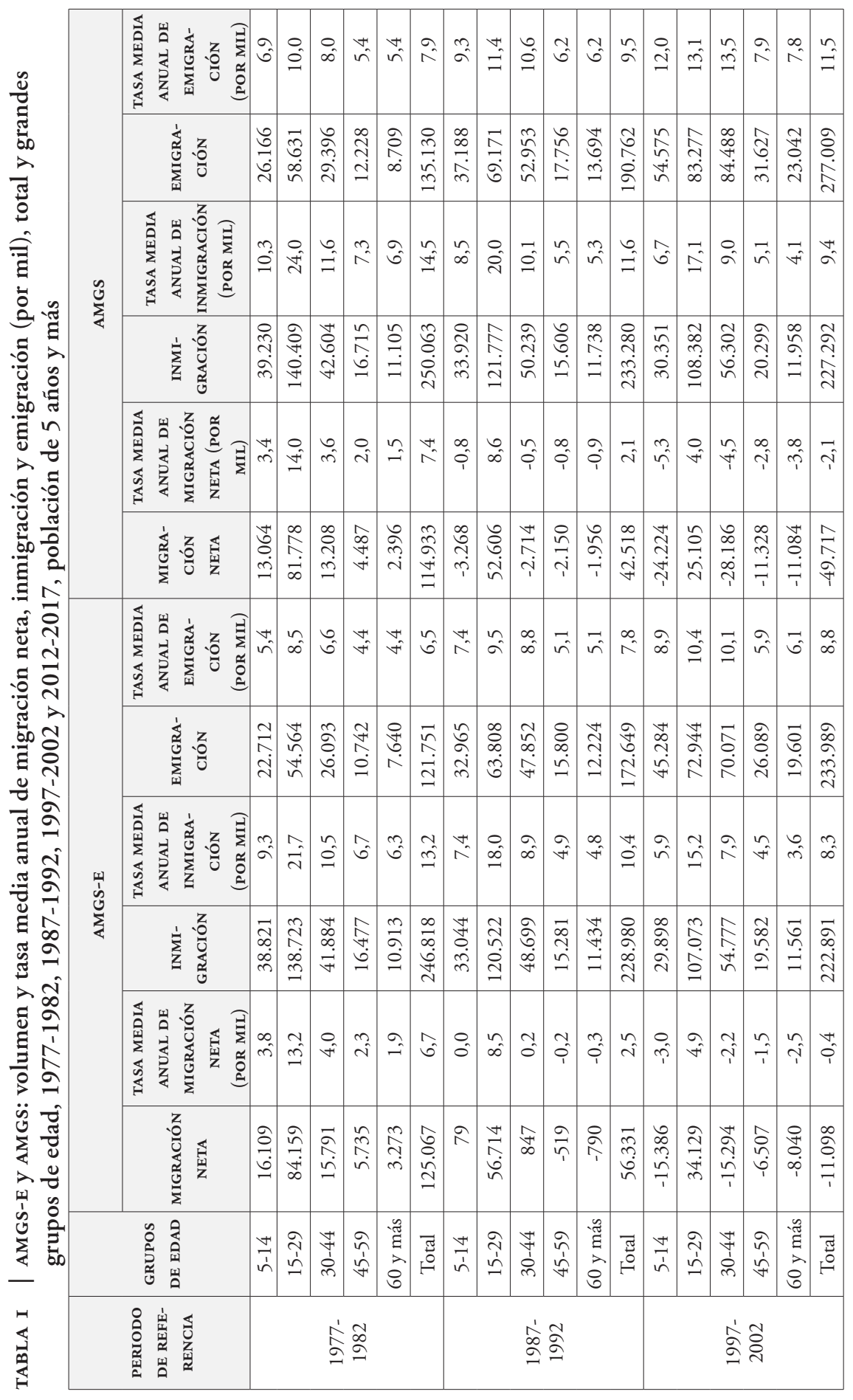




\begin{tabular}{|c|c|c|c|c|c|c|c|}
\hline \multirow{6}{*}{$\sum_{4}^{\infty}$} & 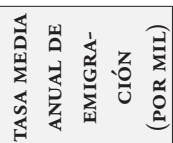 & 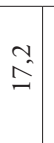 & $\stackrel{\sim}{\simeq}$ & $\begin{array}{c}2 \\
\hat{i}\end{array}$ & $=$ & $\tilde{O}_{0}$ & $\underset{ \pm}{ \pm}$ \\
\hline & 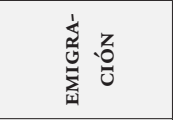 & $\begin{array}{l}\simeq \\
\infty \\
0 \\
\text { ర్ }\end{array}$ & 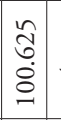 & 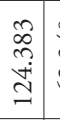 & \begin{tabular}{l|l}
\multirow{+}{*}{} & 1 \\
तై & 1 \\
& 1
\end{tabular} & 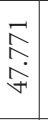 & \begin{tabular}{l}
\multirow{2}{\hat{n}}{} \\
के
\end{tabular} \\
\hline & 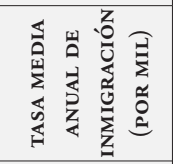 & $\overrightarrow{0}$ & \begin{tabular}{|l|}
$\infty$ \\
$\underbrace{\infty}$
\end{tabular} & $\begin{array}{l}\stackrel{H}{\prime}^{\prime} \\
0\end{array}$ & $\tilde{n}$ & $\hat{\tilde{n}}$ & $\bar{a}$ \\
\hline & 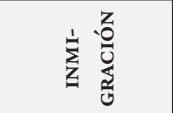 & 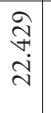 & 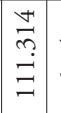 & 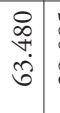 & $\begin{array}{ll} & \\
\infty & 1 \\
0 & : \\
\grave{\lambda} & \end{array}$ & 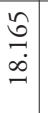 & 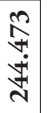 \\
\hline & 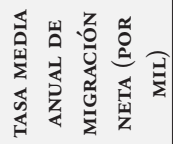 & 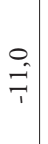 & $\mid \begin{array}{l}\infty \\
0 \\
0\end{array}$ & $\begin{array}{c}0 \\
\hat{\imath}\end{array}$ & $\begin{array}{l}0 \\
\hat{p}\end{array}$ & $\begin{array}{c}\tilde{1} \\
\tilde{\imath}\end{array}$ & $\hat{n}$ \\
\hline & 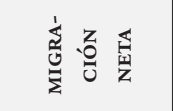 & $\begin{array}{l}\stackrel{0}{2} \\
\text { ळे } \\
\stackrel{+}{1}\end{array}$ & $\mid \begin{array}{l}0 \\
\infty \\
0 \\
0 \\
0\end{array}$ & $\begin{array}{l}m \\
\grave{Q} \\
\stackrel{0}{0}\end{array}$ & 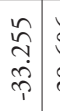 & 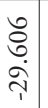 & $\begin{array}{l}\mathbb{R} \\
\stackrel{0}{n} \\
\end{array}$ \\
\hline \multirow{6}{*}{ 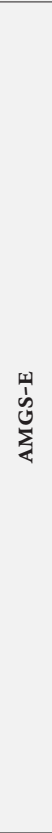 } & 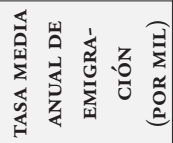 & $\begin{array}{l}0 \\
0 \\
0\end{array}$ & $\hat{n}$ & $\hat{\mathfrak{u}}$ & $\approx$ & $\begin{array}{l}0 \\
\stackrel{n}{n}\end{array}$ & $\hat{\sigma}$ \\
\hline & 离 & $\begin{array}{l}\hat{N} \\
\dot{b} \\
\dot{f}\end{array}$ & 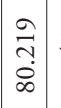 & 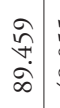 & \begin{tabular}{l|l}
$n$ & \\
$o$ & \\
$\infty$ & \\
+ &
\end{tabular} & $\begin{array}{l}\stackrel{n}{\bumpeq} \\
\hat{n}\end{array}$ & 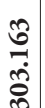 \\
\hline & 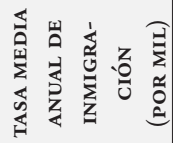 & $\stackrel{\circ}{n}$ & $\hat{\vec{g}}$ & $\tilde{\infty}$ & $\stackrel{m}{m}$ & $\stackrel{m}{m}$ & $\begin{array}{l}0 \\
n^{2}\end{array}$ \\
\hline & 音总 & $\begin{array}{l}\stackrel{\tilde{n}}{\hat{n}} \\
\stackrel{\sim}{\sim}\end{array}$ & $\begin{array}{l}\hat{n} \\
\hat{n} \\
\hat{o} \\
n\end{array}$ & $\begin{array}{l}n \\
\infty \\
\dot{a} \\
\tilde{n}\end{array}$ & $\begin{array}{l}\hat{n} \\
\stackrel{\sim}{v}\end{array}$ & $\begin{array}{l}n \\
\hat{\swarrow} \\
\end{array}$ & 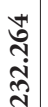 \\
\hline & 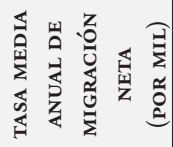 & $\begin{array}{l}\stackrel{0}{i n} \\
\stackrel{i}{i}\end{array}$ & $\begin{array}{c}m \\
m\end{array}$ & $\underset{f}{f}$ & $\stackrel{v}{p}$ & $\vec{y}$ & $\hat{\mathfrak{\imath}}$ \\
\hline & 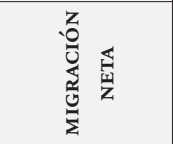 & 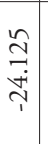 & 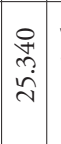 & 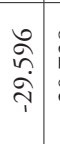 & 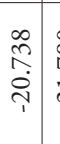 & 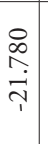 & $\begin{array}{l}\hat{\alpha} \\
\dot{0} \\
\stackrel{R}{1}\end{array}$ \\
\hline & 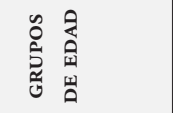 & $\begin{array}{l} \pm \\
\dot{n} \\
n\end{array}$ & $\begin{array}{l}\overrightarrow{\hat{N}} \\
\hat{n}\end{array}$ & 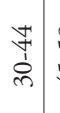 & 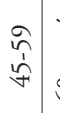 & 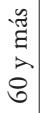 & 胥 \\
\hline & 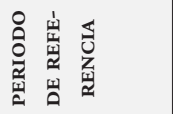 & & & & & & \\
\hline
\end{tabular}


Sus resultados sugieren que, hasta el Censo 1992, la migración neta del AMGs-E no fue selectiva de población con alta educación. ${ }^{4}$ Sin embargo, en el Censo 2002, y sobre todo el de 2017, la tasa de emigración neta de este grupo es más baja que la tasa total, por lo cual la migración sería selectiva del mismo. Estas tasas anticipan efectos de la migración sobre la composición educativa de Santiago cambiantes en el tiempo, los que se estiman con procedimientos novedosos y precisos más adelante en este texto.

En materia de patrones y tendencias, hay similitudes y diferencias con la segmentación etaria. Por un lado, la principal similitud es la inflexión hacia la emigración neta, que también ocurre a fines del siglo pasado (1997-2002). Por el otro lado, la principal diferencia es que no hay uno o más grupos educativos que escapen a esta inflexión, a diferencia de los jóvenes en el caso de la segmentación etaria. En efecto, desde 1997-2002 todos los grupos educativos presentan migración neta negativa, en ambas definiciones de la ciudad.

Durante todo el periodo de observación nunca fue predominante la inmigración de población con baja educación (ocho o menos ańos de escolaridad). Más aún, en 2012-2017, la mayor parte de los inmigrantes al AMGs tenían 13 o más años de escolaridad. Esto no significa forzosamente que los inmigrantes hayan tenido una educación mayor que los no migrantes, duda que se despejará más adelante al examinar el efecto de composición de la inmigración. Pero este hallazgo sí descarta las visiones sobre la "invasión" de población con estudios escasos o nulos a Santiago, al menos desde la década de 1970 en adelante.

Otro hallazgo es el cambio en la heterogeneidad de las tasas de inmigración y emigración según nivel educativo. En 1977-1982 la tasa de inmigración del grupo de alta educación era el doble de la tasa de inmigración del grupo de baja educación, mientras que ese diferencial era incluso más marcado en el caso de la emigración, pues la tasa de emigración del grupo de alta educación era el triple de la tasa de emigración del grupo de baja educación. En cambio, en 2012-2017 el diferencial se amplió para la tasa de inmigración, por cuanto la del grupo de alta educación era más del doble de la del grupo de baja educación, mientras que en el caso de la tasa de emigración el diferencial se estrechó significativamente, pues la del grupo de alta educación fue solo 50\% mayor que la del grupo de baja educación.

Cualquiera sea el caso, la evidencia ya presentada sugiere que la pérdida de atractivo migratorio del AMGs-E y de inflexión hacia la expulsión de población ha tenido diferencias según el nivel educativo de la población y se ha acentuado más en los niveles educativos intermedios y menos en los extremos, sobre todo la población de educación alta. Debido a lo anterior, la pérdida migratoria ha sido más moderada en estos grupos extremos. Por ello, la migración podría estar remodelando el perfil educativo de la población del AMGs-E en un sentido "favorable" para la ciudad; más concretamente, ensanchando el peso relativo de la población con mayor educación, lo que se evaluará empíricamente más adelante en este texto. 


\begin{tabular}{|c|c|c|c|c|c|c|c|c|c|c|c|c|c|c|c|c|c|}
\hline & & 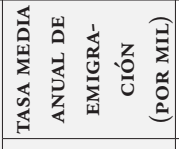 & $\hat{n}$ & $\theta_{0}^{0}$ & $\hat{\infty}$ & $\hat{\Omega}$ & $\stackrel{0}{\sim}$ & $\vec{n}$ & $\stackrel{0}{n}$ & $\stackrel{\infty}{\sigma}$ & $\begin{array}{l}\stackrel{H}{n} \\
\stackrel{n}{n}\end{array}$ & $\sigma^{2}$ & $\stackrel{H}{a}$ & $\stackrel{0}{0}$ & $\Rightarrow$ & $\begin{array}{l}+ \\
\stackrel{n}{n}\end{array}$ & $\cong$ \\
\hline$\lambda$ & & 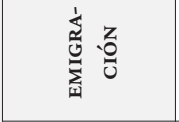 & $\begin{array}{l}\hat{a} \\
\stackrel{\jmath}{j}\end{array}$ & $\begin{array}{l}\vec{T} \\
0 \\
\infty \\
-1\end{array}$ & 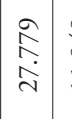 & $\begin{array}{l}\stackrel{g}{G} \\
\stackrel{\Xi}{\Xi}\end{array}$ & 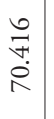 & 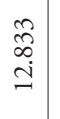 & 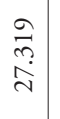 & $\begin{array}{l}\hat{\sigma} \\
\stackrel{+}{+} \\
\text { Fे }\end{array}$ & 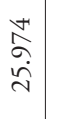 & $\begin{array}{l}\stackrel{n}{\hat{n}} \\
\stackrel{=}{=}\end{array}$ & \begin{tabular}{l}
$\tilde{\sigma}$ \\
\multirow{\sigma}{\sigma}{}
\end{tabular} & $\begin{array}{l}\infty \\
\infty \\
\infty \\
i \\
m\end{array}$ & 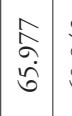 & $\begin{array}{l}\stackrel{\infty}{+} \\
\infty \\
\stackrel{0}{0}\end{array}$ & 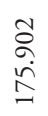 \\
\hline$\hat{\hat{\vec{z}}}$ & $y$ & 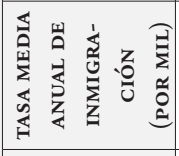 & $\begin{array}{l}0 \\
\dot{0}^{n}\end{array}$ & $\tilde{\sigma}$ & $\vec{m}$ & $\begin{array}{l}0 \\
\stackrel{0}{n}\end{array}$ & $\triangleq$ & $\stackrel{0}{\wedge}$ & $\overbrace{\infty}^{n}$ & $\begin{array}{l}0 \\
0\end{array}$ & $\stackrel{\sim}{\sim}$ & $\stackrel{0}{a}$ & ऊु & $\stackrel{\infty}{0}_{0}^{\infty}$ & $\stackrel{2}{n}$ & $\hat{0}^{0}$ & $\vec{\infty}$ \\
\hline $\begin{array}{c}-\tilde{z} \\
\vdots \\
\lambda \\
0 \\
0\end{array}$ & & 紊总 & 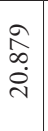 & $\mid \begin{array}{c}\hat{n} \\
\hat{\sigma} \\
\dot{m}\end{array}$ & 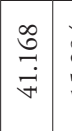 & $\begin{array}{l}\stackrel{+}{\grave{n}} \\
\stackrel{\sim}{=}\end{array}$ & $\begin{array}{l}\infty \\
\stackrel{\infty}{\infty} \\
\infty \\
0 \\
0\end{array}$ & $\begin{array}{l}\vec{尺} \\
\stackrel{i}{i}\end{array}$ & $\begin{array}{l}\stackrel{n}{\forall} \\
\stackrel{\imath}{+}\end{array}$ & $\begin{array}{l}\stackrel{m}{\sim} \\
\stackrel{\infty}{\infty} \\
\stackrel{+}{+}\end{array}$ & 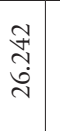 & $\begin{array}{l}\stackrel{a}{0} \\
\infty \\
\stackrel{0}{=}\end{array}$ & $\begin{array}{l}0 \\
\stackrel{1}{\sim} \\
0\end{array}$ & $\begin{array}{l}\overrightarrow{\widetilde{a}} \\
\stackrel{\vec{\lambda}}{ }\end{array}$ & $\mid \begin{array}{l}\hat{\tilde{m}} \\
\stackrel{f}{\tilde{F}}\end{array}$ & 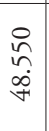 & $\begin{array}{l}\stackrel{+}{N} \\
\stackrel{\sim}{\Xi}\end{array}$ \\
\hline 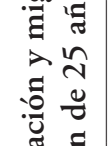 & & 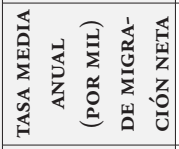 & $\underset{\forall}{*}$ & $\hat{m}$ & $\stackrel{m}{*}$ & $\begin{array}{l}\infty \\
n^{n}\end{array}$ & $\vec{\forall}$ & $\vec{i}$ & : & ô & $\overrightarrow{0}$ & ? & $\tilde{r}$ & $\stackrel{\infty}{\sim}$ & $\stackrel{\infty}{\infty}$ & $\hat{\hat{\imath}}$ & $\stackrel{\vec{r}}{r}$ \\
\hline $\begin{array}{l}0.0 \\
\frac{0}{0} \\
\frac{\pi}{0}\end{array}$ & & 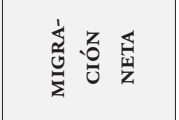 & $\begin{array}{l}\mathbb{S} \\
\infty \\
\infty \\
\infty\end{array}$ & 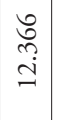 & $\mid \begin{array}{l}\infty \\
\infty \\
n \\
\ddot{n}\end{array}$ & $\underset{m}{m}$ & $\begin{array}{l}\text { ָे } \\
\stackrel{a}{a}\end{array}$ & $\stackrel{\widetilde{2}}{\rightarrow}$ & $\begin{array}{l}\stackrel{*}{0} \\
\text { ì } \\
\text { in }\end{array}$ & $\underset{\infty}{+}$ & $\stackrel{\infty}{i}$ & 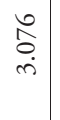 & 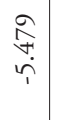 & 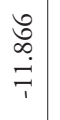 & 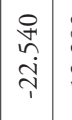 & 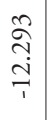 & $\underset{\substack{\sim \\
\sim}}{\stackrel{\sim}{\sim}}$ \\
\hline 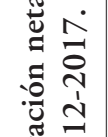 & & 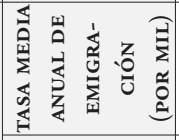 & $\underset{f}{*}$ & $\hat{f}$ & $\hat{\sim}$ & $\hat{\jmath}$ & $\tilde{\sigma}^{2}$ & $\tilde{n}^{2}$ & î & $\stackrel{\forall}{\infty}$ & $\overrightarrow{\mathrm{I}}$ & $\hat{\imath}$ & $\hat{\sigma}$ & $\hat{n}$ & $\begin{array}{l}0 \\
\infty\end{array}$ & $\begin{array}{l}\stackrel{+t}{0} \\
\mathbf{O}^{\prime}\end{array}$ & $\hat{\infty}$ \\
\hline 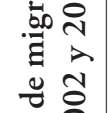 & & 竞 & $\begin{array}{l}\stackrel{0}{\circ} \\
\stackrel{0}{0} \\
\stackrel{0}{0}\end{array}$ & $\begin{array}{l}8 \\
0 \\
0 \\
0 \\
-1\end{array}$ & $\begin{array}{l}0 \\
\stackrel{0}{a} \\
i\end{array}$ & $\begin{array}{l}\infty \\
\stackrel{n}{\Xi} \\
=\end{array}$ & $\begin{array}{l}\vec{\sigma} \\
\stackrel{6}{ } \\
\text { ( }\end{array}$ & $\underset{\substack{+\Xi}}{=}$ & 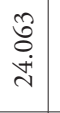 & $\begin{array}{l}\stackrel{0}{2} \\
\stackrel{\infty}{\infty} \\
\stackrel{+}{+}\end{array}$ & 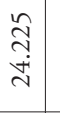 & $\begin{array}{l}\text { } \\
\infty \\
\text { i } \\
0\end{array}$ & $\begin{array}{l}\stackrel{0}{n} \\
\tilde{n}\end{array}$ & $\begin{array}{l}\stackrel{m}{N} \\
\infty \\
\infty\end{array}$ & $\begin{array}{c}n \\
\hat{n} \\
i n\end{array}$ & $\begin{array}{l}n \\
\stackrel{n}{n} \\
\ddot{8} \\
n\end{array}$ & $\begin{array}{l}\stackrel{\sigma}{6} \\
\stackrel{+}{+}\end{array}$ \\
\hline 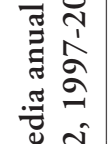 & 我 & 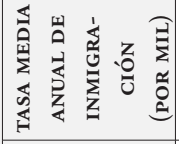 & $\hat{\infty}$ & $\hat{\infty}$ & $\stackrel{\mathfrak{I}}{\simeq}$ & छे & $\hat{0}$ & $\overrightarrow{0}$ & $\stackrel{2}{n}$ & $\stackrel{\circ}{\sigma}$ & $\hat{\mathrm{j}}$ & $\begin{array}{l}0 \\
\infty\end{array}$ & $\stackrel{n}{n}$ & : & $\underbrace{*}_{0}$ & $\stackrel{0}{a}$ & $\vec{n}$ \\
\hline$\approx$ & $\sum_{4}$ & 竞总 & $\begin{array}{l}\text { \&े } \\
\text { ते } \\
\text { ते }\end{array}$ & $\left|\begin{array}{l}n \\
\infty \\
n \\
0 \\
n \\
n\end{array}\right|$ & 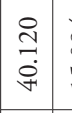 & $\begin{array}{l}\stackrel{+}{a} \\
\stackrel{0}{n}\end{array}$ & $\begin{array}{l}\text { बे } \\
\text { گे }\end{array}$ & $\begin{array}{l}\widetilde{\Xi} \\
\widehat{ָ}\end{array}$ & $\begin{array}{l}\stackrel{0}{\stackrel{2}{2}} \\
\stackrel{2}{2}\end{array}$ & 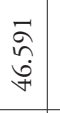 & 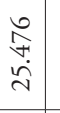 & $\begin{array}{l}\stackrel{0}{\Xi} \\
\stackrel{-}{\rightleftarrows} \\
=\end{array}$ & 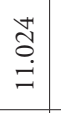 & $\begin{array}{l}\stackrel{0}{n} \\
\stackrel{n}{\sim}\end{array}$ & $\begin{array}{l}a \\
\infty \\
\infty \\
\dot{\forall}\end{array}$ & \begin{tabular}{l}
$\bumpeq$ \\
$\exists$ \\
\multirow{+}{*}{}
\end{tabular} & 苂 \\
\hline 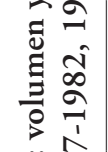 & & 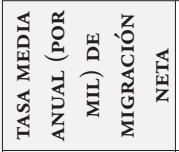 & $\stackrel{\infty}{m}$ & $\stackrel{N}{f}$ & $\stackrel{0}{*}$ & $\stackrel{\sim}{f}$ & $\stackrel{\Downarrow}{\forall}$ & $\stackrel{\infty}{0}$ & $\stackrel{n}{=}$ & $\stackrel{0}{0}_{0}^{\circ}$ & 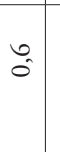 & $\hat{\sigma}$ & $\stackrel{7}{\rightarrow}$ & 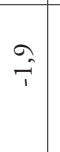 & $\overrightarrow{\hat{\imath}}$ & $\begin{array}{l}\infty \\
0 \\
1\end{array}$ & $\underset{r}{r}$ \\
\hline $\begin{array}{ll}0 & a \\
\sum_{4} & 0 \\
\lambda & 0 \\
1 & 0\end{array}$ & & 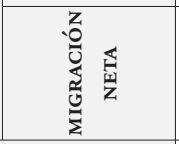 & $\begin{array}{l}0 \\
\infty \\
\infty \\
0 \\
0\end{array}$ & $\left|\begin{array}{l}\infty \\
\infty \\
n \\
+ \\
-1\end{array}\right|$ & 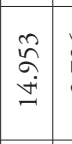 & 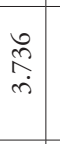 & $\begin{array}{l}\infty \\
\stackrel{2}{\$} \\
\stackrel{+}{+}\end{array}$ & 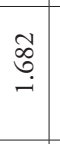 & $\stackrel{m}{=}$ & $\begin{array}{l}\vec{\infty} \\
\stackrel{\sim}{m} \\
\dot{m}\end{array}$ & $\stackrel{\vec{\sim}}{\stackrel{\sim}{\leftrightarrows}}$ & $\stackrel{\widehat{v}}{\stackrel{\Xi}{=}}$ & 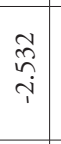 & $\begin{array}{l}\hat{\widehat{\omega}} \\
\infty \\
0 \\
0\end{array}$ & $\hat{n}_{\tilde{\omega}}^{\infty}$ & $\begin{array}{l}\infty \\
\overbrace{0}^{\infty} \\
\dot{+} \\
\dot{i}\end{array}$ & 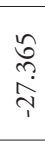 \\
\hline$\sum_{<}^{5} \frac{0}{己}$ & & 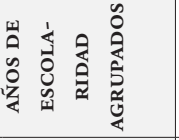 & $\begin{array}{l}+1 \\
0\end{array}$ & $\begin{array}{l}\infty \\
i \\
i\end{array}$ & $\underset{a}{\approx}$ & 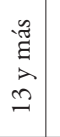 & & d' & $\begin{array}{l}\infty \\
i \\
n\end{array}$ & $\frac{7}{7}$ & 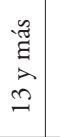 & 륭 & $\stackrel{+}{o}$ & i & $\frac{\sim}{a}$ & 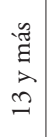 & 丞 \\
\hline & & 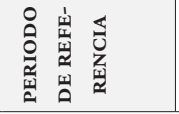 & & & & & & & & & & & & & & & \\
\hline
\end{tabular}




\begin{tabular}{|c|c|c|c|c|c|c|}
\hline \multirow{6}{*}{$\frac{\xi}{4}$} & 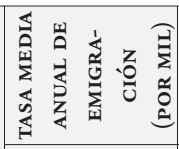 & $\begin{array}{l}\hat{\mathfrak{I}} \\
\mathrm{I}\end{array}$ & $\hat{\widehat{I}}$ & $\underset{+}{ \pm}$ & $\vec{\approx}$ & $\begin{array}{l}0 \\
\stackrel{n}{n}\end{array}$ \\
\hline & 紊㛏 & $\begin{array}{l}\infty \\
\stackrel{0}{0} \\
\stackrel{+}{+} \\
-1\end{array}$ & $\begin{array}{l}\overrightarrow{0} \\
\stackrel{0}{\dot{n}} \\
\tilde{n}\end{array}$ & $\begin{array}{l}\hat{0} \\
\stackrel{+}{=} \\
=\end{array}$ & $\begin{array}{l}\hat{\sigma} \\
\infty \\
\dot{\Xi} \\
=\end{array}$ & $\begin{array}{l}\stackrel{D}{1} \\
\infty \\
\stackrel{\sim}{*}\end{array}$ \\
\hline & 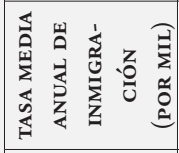 & $\begin{array}{l}\infty \\
\stackrel{f}{*}\end{array}$ & $\begin{array}{l}0 \\
\forall i\end{array}$ & ત્ & 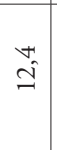 & $\vec{\infty}$ \\
\hline & 光 & \begin{tabular}{l}
\multirow{7}{*}{} \\
$\vec{n}$
\end{tabular} & $\begin{array}{l}\stackrel{+}{n} \\
i n \\
i\end{array}$ & $\begin{array}{l}\infty \\
\stackrel{\infty}{a} \\
\stackrel{+}{+}\end{array}$ & $\begin{array}{l}\hat{D} \\
\infty \\
\dot{\infty} \\
\infty\end{array}$ & 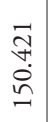 \\
\hline & 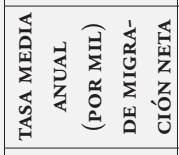 & $\stackrel{\sim}{\stackrel{1}{1}}$ & $\begin{array}{l}n \\
\infty \\
\infty \\
1\end{array}$ & $\overrightarrow{\phi_{1}^{0}}$ & $\begin{array}{l}\infty \\
\hat{f}^{f}\end{array}$ & $\hat{\hat{i}}$ \\
\hline & 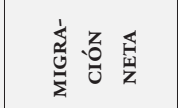 & $\begin{array}{l}0 \\
\infty \\
\infty \\
\infty \\
0 \\
1\end{array}$ & 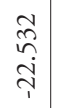 & 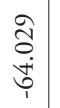 & $\begin{array}{c}\hat{N} \\
\stackrel{\vec{r}}{r}\end{array}$ & $\begin{array}{l}a \\
\hat{\sigma} \\
\stackrel{\Xi}{\sigma}\end{array}$ \\
\hline \multirow{6}{*}{ 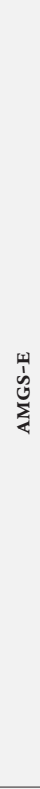 } & 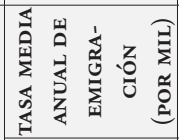 & $\begin{array}{l}n \\
\infty \\
\infty\end{array}$ & $\tilde{\sigma}$ & $\stackrel{\infty}{\sigma^{\prime}}$ & $\Rightarrow$ & $\begin{array}{l}0 \\
0\end{array}$ \\
\hline & 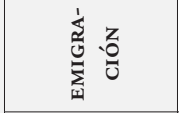 & $\begin{array}{l}\vec{F} \\
\stackrel{\Xi}{\Xi}\end{array}$ & $\begin{array}{l}\infty \\
\stackrel{2}{2} \\
\grave{\lambda}\end{array}$ & \begin{tabular}{l}
\multirow{n}{*}{} \\
$\infty$ \\
$\infty$ \\
$\infty$
\end{tabular} & 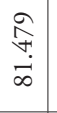 & 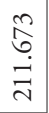 \\
\hline & 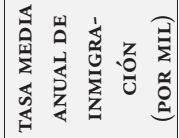 & $\overrightarrow{f^{*}}$ & $\stackrel{\circ}{+i}$ & $\tilde{n}$ & $\stackrel{t}{t}$ & $\hat{\sigma}$ \\
\hline & 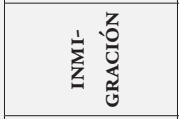 & $\begin{array}{l}\hat{\Xi} \\
\vec{\sigma}\end{array}$ & $\begin{array}{c}\hat{\approx} \\
\grave{\jmath}\end{array}$ & $\begin{array}{c}0 \\
0 \\
\infty \\
i \\
i \\
+\end{array}$ & $\underset{\substack{1\\
}}{ }$ & $\begin{array}{l}\infty \\
\vdots \\
\stackrel{\Xi}{ \pm} \\
\stackrel{\Xi}{I} \\
\end{array}$ \\
\hline & 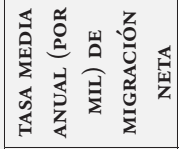 & $\stackrel{\vec{H}}{\stackrel{*}{*}}$ & $\stackrel{\sim}{\sim}$ & $\begin{array}{l}0 \\
\stackrel{+}{+}\end{array}$ & $\hat{i}$ & $\stackrel{m}{\hat{p}_{1}}$ \\
\hline & 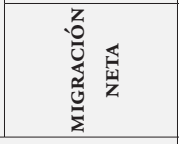 & \begin{tabular}{l}
$\stackrel{+}{n}$ \\
\multirow{n}{0}{} \\
$i$
\end{tabular} & $\begin{array}{l}\hat{\sigma} \\
\hat{\sigma} \\
\div\end{array}$ & $\begin{array}{l}\stackrel{a}{\vdots} \\
\dot{\vec{j}}\end{array}$ & $\tilde{n}_{n}^{n}$ & 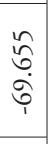 \\
\hline & 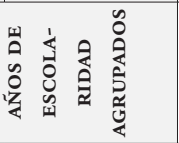 & $\stackrel{+r}{0}$ & $\begin{array}{l}\infty \\
\stackrel{1}{ } \\
n\end{array}$ & $\stackrel{\sim}{a}$ & $\begin{array}{l}\stackrel{n}{घ} \\
\stackrel{2}{2}\end{array}$ & $\stackrel{\widetilde{\tilde{U}}}{\stackrel{0}{0}}$ \\
\hline & 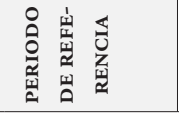 & & & & & \\
\hline
\end{tabular}


Por otra parte. el AMGS-E también concentra una fracción significativa del parque habitacional del país, similar, de hecho, a su peso en la población. Ahora bien, esta similitud se atenúa en los dos últimos censos, pues el parque habitacional del AMGS-E (que pasó de algo más de 1 millón en 1982 a casi 2,4 millones en 2017) representa entre 2 y 3 puntos porcentuales menos que la población, lo que se debe a un diferencial entre la tasa de crecimiento de las viviendas del país (y las urbanas también) y la tasa de crecimiento de las viviendas del AMGs-E. Este último hallazgo es sorpresivo, por cuanto la confluencia de transiciones demográficas (primera y segunda) más avanzadas y de mercados de inmobiliarios más "financiarizados" en Santiago debiera incentivar una construcción habitacional más acelerada en ellas que en el resto del país, pero no ha sido el caso en Chile. Se trata de un asunto que requiere más investigación, pero que se relaciona, probablemente, con la inflexión del atractivo migratorio antes comentada, así como el fenómeno de la segunda vivienda, como se verá (Figura 3).

FIGURA 3 | Chile: porcentaje que representa el AMGs-E en el parque habitacional nacional y urbano, 1982, 1992, 2002 y 2017

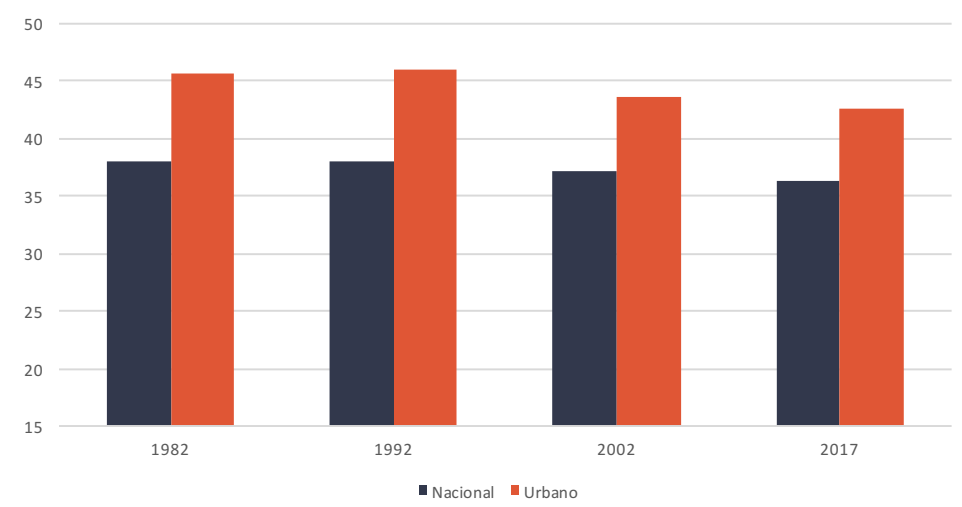

FUENTE: CÁLCULOS PROPIOS. PROCESAMIENTO ESPECIAL DE MICRODATOS CENSALES

Siempre en el tema de vivienda, en el periodo de referencia las viviendas desocupadas y las viviendas ocupadas sin moradores presentes aumentaron mucho más rápido que las ocupadas. Las ocupadas sin moradores presentes fueron las de mayor aumento, lo que se explica básicamente por cambios demográficos y familiares que implican menos gente en casa y más gente trabajando y por ello ausente para el censo, con independencia de que se trate de censos de hecho. A esto se suman crecientes, aunque aún poco significativas, tasas de rechazo a responder al censo (Cabella et al., 2014). De cualquier manera, su aumento fue impresionante: del orden de $800 \%$ (de 31 mil en 1982 a 283 mil en 2017), lo que llevó a un incremento de su peso relativo en el parque desde 1,2\% en 1982 a 4,4\% en 2017. Esta tendencia es un gran desafío para los censos de población, que enfrentan un tozudo 
problema de omisión de población, que se sabe que existe, pero no se logra cuantificar satisfactoriamente.

Por otra parte, las viviendas desocupadas constituyen uno de los asuntos emergentes en materia habitacional, habida cuenta su aumento concomitante con un todavía elevado déficit habitacional. Estas viviendas normalmente no entran en el cálculo de este déficit, porque las razones de su desocupación lo impiden (básicamente son propiedad privada y muchas veces de uso ocasional). En toda América Latina estas viviendas están aumentando, impulsadas por el mercado de segunda vivienda para clases altas y medias, la expansión del mercado de alquiler (que suele implicar desocupación temporal) y la emigración internacional en algunas zonas de países con alta emigración. Por todo eso, las viviendas desocupadas son mucho más frecuentes en áreas rurales, balnearios, zonas turísticas o localidades más bien pequeñas afectadas por alta emigración. La anterior descripción se aplica plenamente en el caso de Chile y el AMGs-E. Los índices de desocupación habitacional en el AMGs han aumentado fuertemente a nivel nacional (335\% entre 1982 y 2017) y más lentamente en el AMGs-E (197\% en el mismo periodo). Por ello, entre 1982 y 2017 el peso de las viviendas desocupadas del AMGs-E dentro del total nacional y el total urbano de viviendas desocupadas se redujo significativamente y en la actualidad es menos de la mitad de su peso demográfico (Figura 4).

FIGURA 4 | Chile: porcentaje que representa el AMGs-E en el parque habitacional de viviendas desocupadas nacional y urbano, 1982, 1992, 2002 y 2017

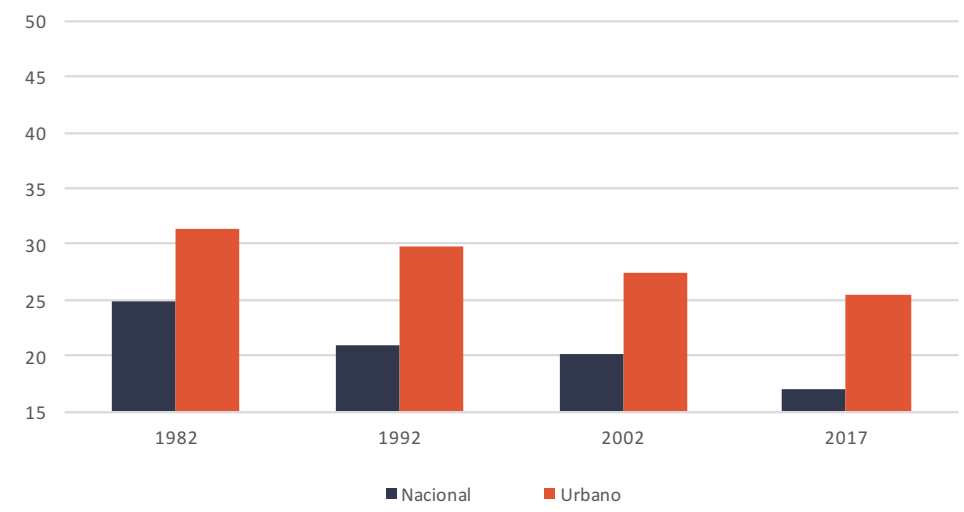

FUENTE: CÁlCULOS PROPIOS. PROCESAMIENTO ESPECIAL DE MICRODATOS CENSALES

\section{Desconcentración genuina o "desconcentración concentrada"}

Aunque ya se comprobó que la migración tiene un efecto "desconcentrador", derivado del signo negativo de la migración neta del AMGs-E desde fines del siglo pasado, puede ocurrir que, finalmente, las pérdidas migratorias se deban al intercambio de la ciudad con zonas o localidades cercanas, cuya creciente articulación podría suponer la constitución de una gran metrópolis difusa o una ciudad-región o, al 
menos, un área de influencia ampliada de Santiago. Este fenómeno también puede corresponder a lo que la literatura ha denominado "desconcentración concentrada", como se mencionó en la sección de antecedentes. Entonces, su eventual ocurrencia pondría en jaque las narrativas de la desconcentración sugerida por la emigración neta del AMGs-E.

Se trata de una discusión teórica muy en boga por los debates sobre las nuevas formas metropolitanas, crecientemente fragmentadas y sobre todo difusas (Brenner, 2014; Buzai, 2016), y la evidencia en algunos países de la región de flujos masivos de salida desde las grandes ciudades hacia localidades de su entorno que, si bien no son suburbios en sentido estricto, se hallan estrechamente vinculadas a la gran ciudad y podrían formar parte de una misma megápolis (Cunha, 2018).

Definir los límites de una ciudad es complejo y en general controversial. Definir los límites de un área metropolitana extendida o difusa, ciudad-región, megápolis o área de influencia a gran escala, es incluso más complejo y debatible. No hay metodologías estándares o fuentes para implementarlas en la región, más allá de excepciones nacionales en México y Brasil, por ejemplo (CEPAL, 2014; RodríguezVignoli, 2019b).

Cualquiera sea el caso, es probable que respecto de Santiago haya consenso en que una eventual megápolis podría extenderse en un radio de hasta 100 kilómetros a la redonda de su Plaza de Armas, lo cual le permitiría incorporar al aglomerado Vińa-Valparaíso (con todas sus localidades), a Quintero, al puerto de San Antonio y la franja costera próxima hasta Valparaíso por el oeste y noroeste, a Los Andes y San Felipe por el nororiente, y a Rancagua por el sur. Entonces, usando los datos de las matrices de ciudades de MiALC (https://celade.cepal.org/bdcelade/mialc/), se revisaron los flujos del AMGS-MIALC con las ciudades antes mencionadas.

Los cálculos efectuados permiten concluir que en el periodo 2012-2017 hay una desconcentración demográfica genuina y no una "desconcentración concentrada". Lo anterior, porque una parte importante de los flujos se dirige a regiones alejadas del Amgs-mialc. Adicionalmente, la pérdida que registra AMgs-mialC no se compensa con la ganancia de las ciudades que podrían formar parte de la eventual "ciudad-región o macrorregión de Santiago". Es cierto que la mayoría de estas ciudades registra saldos migratorios positivos, y en todas ellas los saldos bilaterales con Santiago les son favorables, como se aprecia en la Tabla 3, donde sobresale San Antonio (que incluye la seguidilla de balnearios desde Cartagena hasta Algarrobo) con un saldo bilateral de 11.614 personas. Por su parte, entre las ciudades "lejanas" que captan los flujos desde Santiago sobresalen La Serena (470 km al norte), Temuco (690 km al sur) y Puerto Montt (1.032 km al sur), y a otras más novedosas, como Villarrica $(760 \mathrm{~km}$ al sur) (véase mialc). Por cierto, lejanía en modo alguno significa automáticamente saldo bilateral negativo para Santiago, como lo revelan las ciudades extremas del norte (Arica e Iquique), Concepción en el sur (Tabla 3) o la mayoría de las ciudades mineras del norte (véase mialc, no se presentan en la tabla). Estas últimas, contra la intuición generalizada sobre su atractivo permanente, han tendido a perder población por migración interna, lo que puede deberse a fenómenos emergentes como la conmutación a larga distancia (Atienza \& Aroca, 2012; Rowe \& Bell, 2017) o a la sustitución por migración internacional. 
Un hallazgo llamativo es que la pérdida migratoria de Santiago mialc se debe principalmente a su intercambio con la categoría "otros" de MIALC, que agrupa a todas las comunas que no tienen o no forman parte de una ciudad de 20.000 habitantes o más. El saldo bilateral de este intercambio es negativo para Santiago MIALC en algo más de 41.000 efectivos en 2012-2017 y en torno a 8.200 en 1997 2002 (Tabla 3). Dado que estas comunas "no citadinas" pueden distribuirse a través de todo el país y podrían estar cerca de Santiago y con ello abonar a la hipótesis de la "desconcentración concentrada", en la Tabla 4 se hace un ejercicio definitivo, en el cual se usa toda la Región Metropolitana como aproximación a Santiago, y como área de influencia o de expansión difusa a tres regiones que se denominan "cercanas" (Valparaíso, O’Higgins y Maule, que se extienden desde 170 kilómetros al norte de Santiago hasta 300 kilómetros al sur); las otras 12 regiones se clasifican como "lejanas". Los resultados sugieren que en torno a dos tercios de la pérdida migratoria de la Región Metropolitana se deben a flujos con las regiones cercanas, pero que un tercio se debe a flujos con regiones lejanas, ratificando que la migración entrańa una desconcentración real, aunque recortada respecto de la cifra inicial de emigración neta de la RM o del AMGs-E.

Cabe mencionar que este escenario era diferente a inicios de siglo, pues el Censo 2002 indicaba que Santiago MIALC ganaba en los intercambios bilaterales con la mayoría de las otras ciudades y que toda la emigración neta de la RM se explicaba por el intercambio bilateral con las regiones cercanas. En tal sentido, hasta fines del siglo pasado la migración interna podría haber empujado una suerte de "desconcentración concentrada" a gran escala, pero actualmente las cifras sugieren que esta fase ya ha quedado atrás y que la migración promueve una desconcentración demográfica real, aunque de magnitudes muy inferiores a la que se deriva de su emigración neta. 


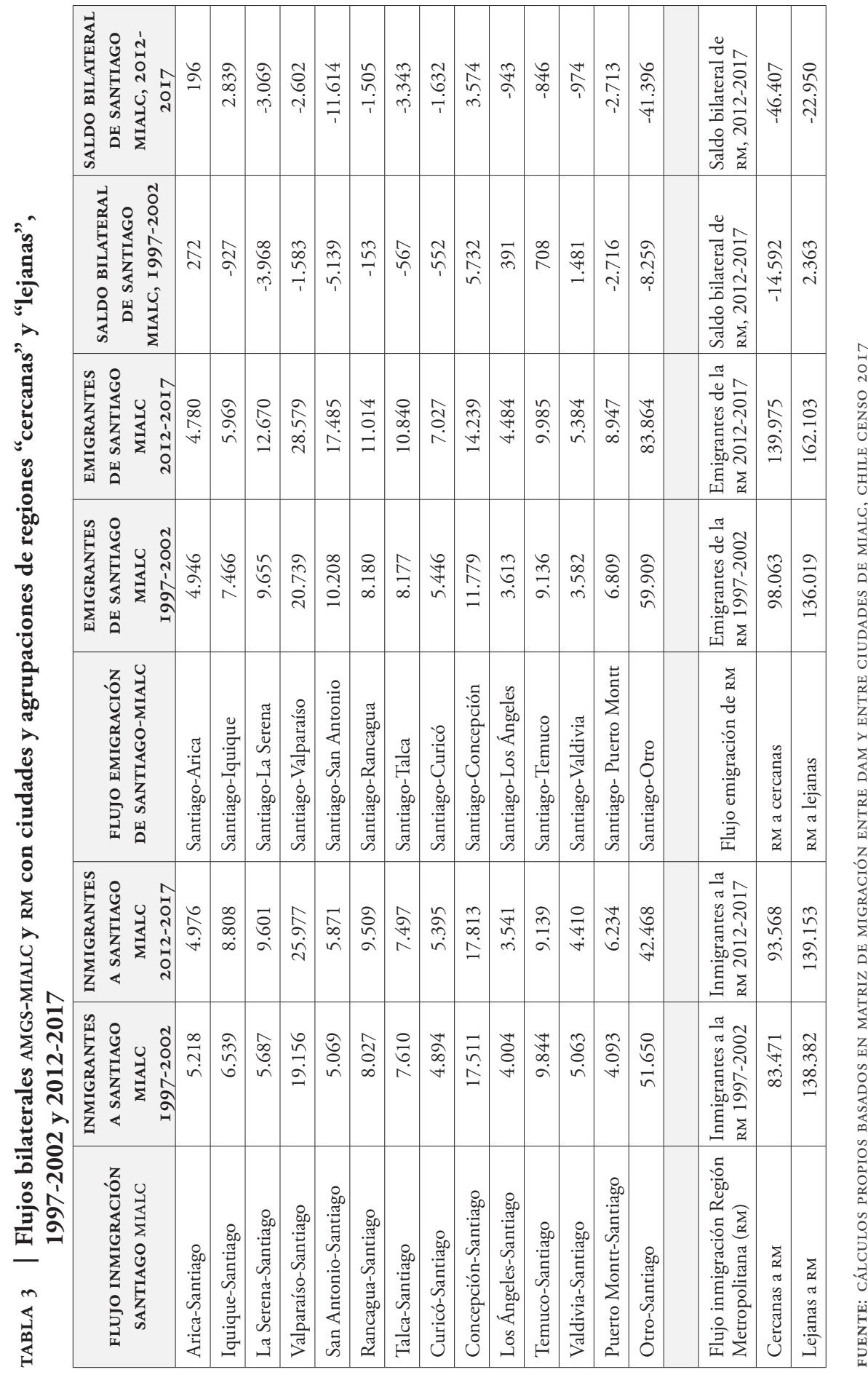


Cualquiera sea el caso, esta tendencia hacia la desconcentración de los flujos migratorios, así como la profundización del carácter expulsor de Santiago, todavía generan dudas y polémicas, sea por datos o sea solo por imágenes e inercia. Parte de estas dudas viene de lecturas alternativas, como la planteada por RodríguezVignoli y Rowe (2018b), que, reconociendo la desconcentración demográfica (vía el denominado "efecto crecimiento"), encuentran resultados diferentes si se considera la calificación y algunas características de las personas (vía el denominado "efecto de composición”). Por ello, este asunto será retomado en la siguiente sección para finalizar el análisis empírico.

Por otra parte, cabe consignar que la tendencia "desconcentradora demográfica" anterior atañe solo a la migración interna y no incluye la migración internacional. Esta última tuvo magnitudes significativas y crecientes en los periodos de referencia de la migración reciente de los últimos dos censos -1997-2002 y 2012-2017 (INE, 2018) - y, como destino preferencial, al AMGS-E. En efecto, en el periodo 20122017 se registró una masiva inmigración desde otros países, principalmente latinoamericanos y caribeńos, a Chile. Su patrón de asentamiento, al menos inicial, no fue aleatorio, pues se concentró claramente en la ciudad de Santiago (67\% en el AMGs-E. Véase Rodríguez-Vignoli y Rowe, 2018b, p. 62). En este sentido, los más de 300.000 inmigrantes internacionales del periodo 2012-2017 residentes en el AMGS-E introducen un mayúsculo signo de interrogación sobre el atractivo de la ciudad, pues son casi cinco veces el saldo migratorio negativo interno. Así las cosas, pese a su emigración interna neta, el crecimiento demográfico del AMGS-E recibe un impulso adicional por la inmigración internacional. Esto resulta clave para el aumento de la concentración de la población en esta ciudad, como se vio con el indicador de porcentaje de la población total que representa el AMGS-E (Figura 2).

\section{Efectos de la migración interna sobre la composición etaria y socioeconómica del AMGS-E}

Las tablas 4 y 5 exponen el efecto relativo de la migración sobre la estructura etaria y la estructura educativa, respectivamente, usando las categorías antes utilizadas para agrupar edad y años de escolaridad. Sus resultados sintetizan gran cantidad de información previa y estiman cuantitativamente y con precisión la magnitud de un efecto anticipado previamente (al menos en su signo) por la comparación de las tasas de migración neta de los grupos etarios y de los grupos educativos, con las de sus respectivos totales.

En esa línea, los resultados de la Tabla 4 no son sorpresivos, pues se podían prever a partir del mayor atractivo del AMgs-E para los jóvenes en el Censo 1982, junto con el contrapunto entre el AMGS-E atractiva para los jóvenes y expulsora para el resto de las edades que revelaron los censos de 1992, 2002 y 2017 (Tabla 1). La novedad estriba en la estimación numérica del efecto. Llamativamente, este ha sido más bien constante durante el periodo de observación, pese a la significativa merma 
de la intensidad del atractivo migratorio del AMGs-E para todas las edades. ${ }^{5}$ De esta manera, en los últimos cuarenta años, la migración ha aumentado el porcentaje de jóvenes del AMGS-E en torno a un $3 \%{ }^{6}$ en cinco ańos (periodos de referencia de la migración en cada censo) y ha visto reducido el porcentaje de los otros grupos de edad entre un $1 \%$ y un 2,5\%, dependiendo del grupo y el periodo de referencia (o censo).

Ahora bien, salvo los menores de 15 años (en rigor 5 a 14 años), todos los otros grupos de edad (excluyendo los jóvenes) presentan una moderación del efecto reductor que ejerce la migración sobre su porcentaje en el AMGs. Por ello, el impacto remodelador de la migración interna sobre la estructura etaria de la ciudad se atenúa durante el periodo de observación, aunque evidentemente persiste, como ya se indicó, su efecto rejuvenecedor. Cualquiera sea el caso, el AMGs-E se suma al resto de ciudades latinoamericanas que sistemáticamente experimentan un efecto rejuvenecedor por la migración (Rodríguez-Vignoli, 2017, 2019b; RodríguezVignoli \& Rowe, 2018b). Como ya se indicó, los factores determinantes de este hecho estilizado aún requieren mayor indagación. Y lo mismo pasa con sus consecuencias, porque aún no están bien documentados los impactos socioeconómicos, habitacionales, culturales y urbanos en general de este fenómeno. Por otra parte, la Tabla 4 deja en evidencia que este rejuvenecimiento del AMGS-E es a expensas del angostamiento del porcentaje de jóvenes en el resto del país, como podía preverse.

Respecto al efecto sobre la estructura educativa, los resultados (Tabla 5) muestran que hasta la década de 1990 el AMGs-E registró un comportamiento como el previsto en la literatura sobre la migración de las grandes ciudades de América Latina (Rodríguez-Vignoli \& Rowe, 2018a), pues el efecto de la migración era reductor del porcentaje de personas con alta educación, mientras elevaba la proporción de otros grupos con distintos niveles educativos, los que variaban según el censo. Pero eso cambia desde finales de la década de 1990 y definitivamente se invierte en el siglo xxi. El Censo 2002 captura el efecto polar comentado en la discusión conceptual, por cuanto la migración aumenta simultáneamente el porcentaje de población con muy baja educación ( 0 -4 años de escolaridad) y el porcentaje de población con educación alta (13 años o más de escolaridad). El Censo 2017, por su parte, muestra un cambio importante: la migración solo eleva el porcentaje de población con alta educación en el AMGs-E (1,3\% en cinco años), mientras reduce el porcentaje de los otros tres grupos medidos según niveles educativos. Esto último ratifica la conclusión efectuada con base en los datos de saldo y tasa de migración neta: sin lugar a duda el AMGS-E ha devenido expulsora en los últimos veinte años, pero la migración tuvo una inflexión cualitativa concomitante y ahora fortalece la base de recursos humanos calificados, que en el pasado más bien diluía.

5 Siendo el atractivo migratorio expresado a través de la tasa de migración neta de cada grupo, uno de los dos componentes del efecto de la migración sobre la estructura etaria (Rodríguez-Vignoli, 2019b; Rodríguez-Vignoli \& Rowe, 2018a).

6 Cabe recordar que no se trata de puntos porcentuales, sino de cambio relativo, expresado por cien, del porcentaje de jóvenes y de los otros grupos de edad. 

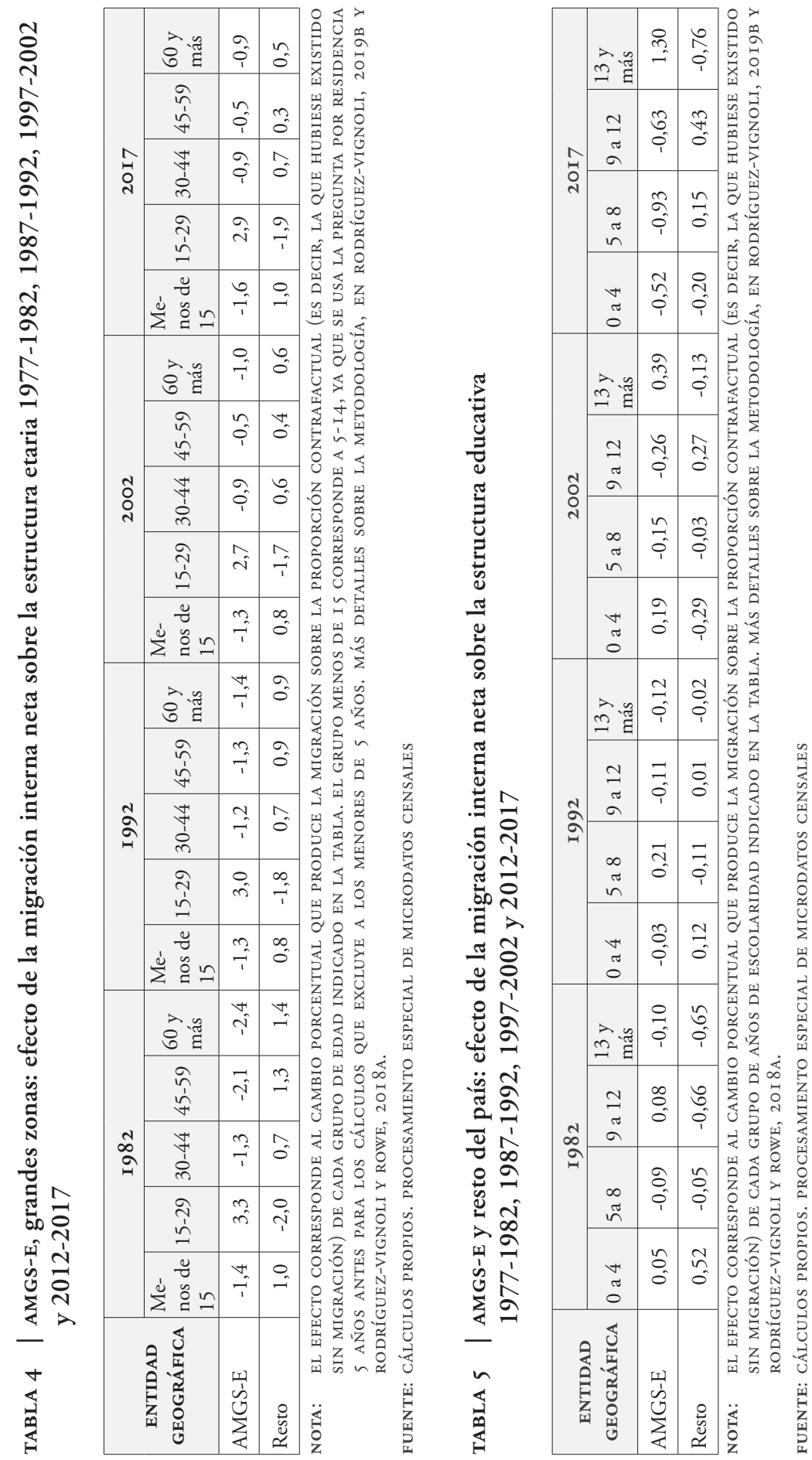


\section{Reflexiones finales}

Durante el periodo de observación, hay indicios de una tendencia hacia la desconcentración demográfica del país, como lo sugieren la pérdida de gravitación del AMGs-E en la población urbana y el parque habitacional (viviendas ocupadas y desocupadas) nacional. Sin embargo, otros indicadores apuntan en la dirección contraria, pues la población del AMGS-E aumentó sostenidamente su peso relativo en la población total y mantuvo su peso relativo en el total de viviendas ocupadas del país. Esta ambigüedad impide caracterizar la tendencia demográfica del sistema de asentamientos humanos de Chile como una desconcentración franca. Pero al menos permite descartar que el país se encuentre en un proceso de aumento de la concentración demográfica. Cualquiera sea el caso, es claro que las expectativas desconcentradoras derivadas del modelo de desarrollo de exportación primaria para los mercados globales, así como de un conjunto de políticas y planes que han apuntado genéricamente hacia ese objetivo, no se han cumplido. De hecho, Chile sigue siendo un país altamente concentrado, al menos en términos demográficos, en su ciudad principal, Santiago.

No obstante lo anterior, desde la migración interna sí hay seńales de desconcentración. En particular, de acuerdo con el último censo de 2017 (periodo 2012 2017 para la migración), se consolidó la emigración neta del AMGS-E y de la Región Metropolitana, que ya había sido detectada por el Censo 2002. Además, un análisis más detallado de los flujos, posibilitado por la base de datos MIALC, muestra que: i) casi un tercio de la pérdida de la Región Metropolitana se debe al intercambio con regiones lejanas, lo que permite descartar que tras la emigración neta haya solo "desconcentración concentrada"; ii) la pérdida del AMGS-E con los municipios sin ciudades se incrementó significativamente $y$, de hecho, este segmento del sistema de asentamientos humanos, históricamente expulsor, devino el más atractivo, lo que es una gran novedad, sin causas claras todavía y que recién comienza a reconocerse y estudiarse (Rodríguez-Vignoli, 2019a).

La pérdida de atractivo migratorio, la inflexión hacia la emigración neta y el alza de la tasa de emigración pueden considerarse señales de deterioro relativo de las condiciones y opciones de vida en el AMGs-E. Pero los indicadores básicos y tradicionales de las condiciones y opciones de vida, como los índices de desocupación, de ingresos y de pobreza, no parecen haber experimentado grandes retrocesos relativos durante lo que lleva el siglo (Fuentes et al., 2017; Rodríguez-Vignoli, 2019b). Por ello, tal vez las fuerzas expulsoras pudieran estar en varios problemas que afligen a la ciudad desde hace tiempo -desde la contaminación a la congestión vehicular-, así como en los crecientes costos de vivir en ella, en particular de alquilar o comprar viviendas, sobre todo las localizadas en zonas de buena conectividad y alta demanda de residencia. Sin embargo, estas fuerzas expulsoras no parecen tener el mismo efecto sobre la migración internacional, la que durante el periodo de referencia del último censo tuvo un gran aumento y una marcada concentración en el AMGS-E, contribuyendo a la expansión de la fuerza de trabajo y de la producción en general, por su alta participación laboral y sus mayores niveles de calificación (INE, 2018; Rodríguez-Vignoli, 2019a). 
Por otro lado, estas fuerzas expulsivas actúan de manera diferenciada según edad. De hecho, el AmGs-E aún resulta atractiva para los jóvenes. En este sentido Santiago se asemeja a la gran mayoría de las ciudades de la región, que mantienen un importante flujo de inmigrantes jóvenes, probablemente atraído por las mayores opciones educacionales, la diversidad del mercado de trabajo y el dinamismo de empleos típicamente juveniles, una oferta inmobiliaria de alquiler más funcional para este grupo etario (más allá de su elevado costo) y una mayor oferta de actividades culturales y recreativas, dimensión importante para los jóvenes (Rodríguez-Vignoli, 2019a). En cambio, el AMGs-E es particularmente expulsora de familias en fase de crianza, las que enfrentan un escenario más hostil por los tiempos de transporte, las tensiones de la dinámica metropolitana y los costos de las viviendas familiares. Cualquiera sea el caso, este contrapunto entre atracción de jóvenes y expulsión de las restantes edades tiene un efecto evidente sobre la estructura etaria, cual es que la migración rejuvenece (es decir, aumenta el porcentaje de jóvenes o población de 15 a 29 años de edad) al AMGs-E. Con ello, refuerza el peso relativo de este segmento etario, el más expuesto a la innovación y a la tecnología y el más adaptable y flexible para enfrentar el cambio continuo de la economía y la sociedad actuales. En tal sentido, más allá de la pérdida de efectivos derivada de la migración, el AMGs-E mantiene una ganancia del peso relativo de los jóvenes que, probablemente, contribuye al dinamismo económico y sociocultural de la ciudad.

Algo similar ocurre con la población de mayor educación. Claro que, en este caso, el AMGs-E también es expulsora de la misma, pero en menor grado que los otros grupos educativos y, por ello, su efecto de composición final es aumentar el peso relativo de esta población, reforzando el porcentaje de recursos humanos calificados.

De esta manera, la migración interna tiene, finalmente, dos caras en el caso del AMGS-E. De una parte, muestra algún tipo de deterioro o encarecimiento relativo del AMGS-E, que desata fuerzas expulsoras y lleva a una fracción creciente de la población a emigrar, una fracción significativa a zonas y localidades cercanas, pero una parte no menor a regiones y localidades más allá de los límites de "lo cercano". Pero por otra, tiene efectos de composición etaria y educativa que resultan funcionales, en principio, para la economía y el desarrollo sociocultural de las ciudades. De esta forma, la migración puede estar, finalmente, fortaleciendo al AMGS-E.

\section{Referencias bibliográficas}

Aguilar, A. (Coord.). (2006). Las grandes aglomeraciones y su periferia regional. Experiencias en Latinoamérica y España. Instituto de Geografía, Universidad Nacional Autónoma de México (unam). Porrúa.

Aguilar, A. \& Escanilla, I. (Coords.). (2011). Periurbanización y sustentabilidad en grandes ciudades. Instituto de Geografía, Universidad Nacional Autónoma de México (unam). Porrúa. 
Atienza, M. \& Aroca, P. (2012). Concentración y crecimiento en Chile: una relación negativa ignorada. EURE, 38(114), 257-277. http://dx.doi.org/10.4067/S025071612012000200010

Bilsborrow, R. (2016). Concepts, definitions and data collection approaches. En M. J. White (Ed.), International Handbook of Migration and Population Distribution. Springer, International Handbooks of Population 6. https://doi.org/10.1007/978-94-0177282-2_7

Brenner, N. (2014). Teses sobre a urbanizaçấo. e-Metropolis. Revista electrônica de estudos urbanos e regionais, 19(5), 6-26. http://emetropolis.net/artigo/146?name=teses-sobrea-urbanizacao

Buzai, G. (2016). Urban models in the study of Latin American cities. Universität Innsbruck [en línea]. https://www.researchgate.net/publication/305433398_Urban_Models_in_ the_Study_of_Latin_American_Cities

Cabella, W., Macadar, D., Ruiz, M. \& Rodríguez, J. (2014). Los datos demográficos. Alcances, limitaciones y métodos de evaluación. CEPAL, Serie Manuales n. 82 [LC/L.3906]. https://repositorio.cepal.org/handle/11362/37145

Celade (Centro Latinoamericano y Caribeño de Demografía) \& prolap (Programa Latinoamericano de Actividades de Población). (1998). Demografía I. Universidad Nacional Autónoma de México (UnAm).

Chávez, A., Acuña, M., Barquero, J., Cunha, J., Macadar, D., Rodríguez, J. \& Sobrino, J. (2016). Migración interna y cambios metropolitanos: ¿qué está pasando en las grandes ciudades de América Latina? Revista Latinoamericana de Población (RELAP), 10(1), 7-41. http://revistarelap.org/ojs/index.php/relap/article/view/137/145

cepal (Comisión Económica Para América Latina y El Caribe). (2012). Población, territorio $y$ desarrollo sostenible. CePAL, Celade-División de Población [LC/L.3474(CEP.2/3)]. https://repositorio.cepal.org/bitstream/handle/11362/22450/1/S1200093_es.pdf

Cepal (Comisión Económica Para América Latina y El Caribe). (2014). Panorama social de América Latina 2014. CEPAL [LC/G.2635-P]. https://repositorio.cepal.org/bitstream/ handle/11362/37626/S1420729_es.pdf

Cunha, J. (Org.). (2018). Dinâmica demográfica e socioespacial no Brasil Metropolitano: convergências e especificidades regionais. EDUFSCAR (Editorial Universidade Federal de São Carlos).

Cunha, J. \& Rodríguez, J. (2009). Urban growth and mobility in Latin America. En S. Cavenaghi (Org.), Demographic transformations and inequalities in Latin America. Historical trends and recent patterns. Latin American Population Association, Serie Investigaciones, n. ${ }^{\circ}$ 8, 25-63. http://www.alapop.org/alap/SerieInvestigaciones/ InvestigacionesSI1aSi9/DemogTransformations_ParteI-1.pdf

De Mattos, C. (2010). Globalización y metamorfosis metropolitana en América Latina: de la ciudad a lo urbano generalizado. Revista de Geografia Norte Grande, 47, 81-104. http:// dx.doi.org/10.4067/S0718-34022010000300005

De Mattos, C. \& Hidalgo, R. (Eds.). (2007). Santiago de Chile: Movilidad espacial y reconfiguración metropolitana. Instituto de Estudios Urbanos y Territoriales, Pontificia Universidad Católica de Chile. Colección EURE Libros \& Serie GEOlibros n. ${ }^{\circ} 8$. 
De Mattos, C., Fuentes, L. \& Link, F. (2016). Tendencias recientes del crecimiento metropolitano en Santiago de Chile. ¿Hacia una nueva geografía urbana? Revista INVI, 29(81), 193229. http://www.revistainvi.uchile.cl/index.php/INVI/article/view/837/1148

Dureau, F., Dupont, V., Lelièvre, E., Lévy, J. \& Lulle, T. (Coords.). (2002). Metrópolis en movimiento. Una comparación internacional. Alfaomega.

Dureau, F., Lulle, T., Souchaud, S. \& Contreras, Y. (Dirs.). (2014). Mobilités et changement urbain. Bogotá, Santiago et São Paulo. Presses Universitaires de Rennes.

Florida, R. (2005). Cities and the Creative Class. Routledge.

Frey, W. H. (1987). Migration and depopulation of the metropolis: regional restructuring or rural renaissance. American Sociological Review, 52(2), 240-257. https://doi. org/10.2307/2095452

Fujita, M. \& Krugman, P. (2004). The new economic geography: Past, present and the future. Papers in Regional Science, 83(1), 139-164. https://doi.org/10.1007/s10110-0030180-0

Fujita, M., Krugman, P. \& Venables, A. (2000). The spatial economy: Cities, regions, and international trade. The MIT Press.

Fuentes, L., Mac-Clure, O., Moya, C. \& Olivos, C. (2017). Santiago de Chile: ¡ciudad de ciudades? Desigualdades sociales en zonas de mercado laboral local. Revista de la CEPAL, (121), 93-109. http://repositorio.cepal.org/handle/11362/41146

Galetovic, A. \& Jordán, P. (2006). Santiago: ¿Dónde estamos? ¿Hacia dónde vamos? Estudios Públicos, (101), 87-145. https://www.cepchile.cl/santiago-donde-estamos-haciadonde-vamos/cep/2016-03-04/093914.html

Harvey, D. (2014). Diecisiete contradicciones y el fin del capitalismo. Instituto de Altos Estudios Nacionales del Ecuador (IAEN).

Ingram, G. (1998). Patterns of metropolitan development: What have we learned? Urban Studies, 35(7), 1019-1035. http://journals.sagepub.com/doi/abs/10.1080/0042098984466

Instituto Nacional de Estadísticas de Chile (INE). (2018). Características de la inmigración internacional en Chile, Censo 2017.

Pacione, M. (2009). Urban geography. A global perspective. Routledge.

Rees, P., Bell, M., Duke-Williams, O. \& Blake, M. (2000). Problems and solutions in the measurement of migration intensities: Australia and Britain compared. Population Studies, 54(2), 207-222. https://doi.org/10.1080/713779082

Ribeiro, L. (2015). O futuro das metrópoles. desigualdades e governabilidade. Letra Capital.

Roberts, B. \& Wilson, R. (2009). Urban segregation and governance in the Americas. Palgrave and Macmillan.

Rogers, A. \& Castro, L. (1982). Patrones modelo de migración. Demografía y Economía, 16(3), 267-327. http://dx.doi.org/10.24201/edu.v16i03.529

Rodríguez, A. \& Sugranyes, A. (Eds.). (2005). Los con techo. Un desafio para la politica de vivienda social. Ediciones SUR, Colección Estudios Urbanos. http://www.sitiosur.cl/r. php?id $=81$

Rodríguez-Vignoli, J. (2009). La captación de la migración interna mediante censos de población: la experiencia de la ronda de 2000 y sus lecciones para la ronda de 2010 en América Latina y el Caribe. Notas de Población, 88, 63-95. http://repositorio.cepal. org/handle/11362/12847 
Rodríguez-Vignoli, J. (2013). La migración interna en las grandes ciudades en América Latina: efectos sobre el crecimiento demográfico y la composición de la población. Notas de Población, 96, 53-104. https://repositorio.cepal.org/handle/11362/12909

Rodríguez-Vignoli, J. (2017). Migración interna y asentamientos humanos en América Latina y el Caribe (1990-2010). CepaL, Serie Población y Desarrollo n. ${ }^{\circ} 121$ [LC/Ts.20 I7/I I 5 ]. https://repositorio.cepal.org/handle/11362/42538

Rodríguez-Vignoli, J. (2019a). Migraciones internas en Chile, 1977-2017: continuidad y cambio. CEPAL, Serie Población y Desarrollo n. ${ }^{\circ} 126$ [LC/TS.2019/75]. https://repositorio.cepal. org/handle/11362/45047

Rodríguez-Vignoli, J. (2019b). El efecto de la migración interna sobre la estructura y las disparidades etarias en las grandes ciudades de América Latina (Tesis doctoral inédita). Universidad Nacional de Córdoba, Argentina. https://rdu.unc.edu.ar/handle/11086/11654

Rodríguez-Vignoli, J., Abarca, C., Becker, I. \& Páez, K. (2017). ¿Perdió el Área Metropolitana del Gran Santiago su atractivo? Sí, pero no. Un examen basado en datos y procedimientos novedosos para la estimación de la migración interna y sus efectos durante el periodo 1977-2013. EURE, 43(128), 5-30. http://dx.doi.org/10.4067/ S0250-71612017000100001

Rodríguez-Vignoli, J. \& Espinoza, D. (2012). Recuperación del atractivo migratorio metropolitano en el periodo 2004-2009: ¿‘factores exógenos o endógenos? Revista de Geografia Norte Grande, 51, 95-113. http://dx.doi.org/10.4067/S071834022012000100006

Rodríguez-Vignoli, J. \& Rowe, F. (2018a). How is internal migration reshaping metropolitan populations in Latin America? A new method and new evidence. Population Studies, 72(2), 253-273. https://doi.org/10.1080/00324728.2017.1416155

Rodríguez-Vignoli, J. \& Rowe, F. (2018b). Efectos cambiantes de la migración sobre el crecimiento, la estructura demográfica y la segregación residencial en ciudades grandes: el caso de Santiago, Chile, 1977-2017. cepal Serie Población y Desarrollo, n. ${ }^{\circ} 125$ [LC/TS.2018/110]. https://repositorio.cepal.org/bitstream/handle/11362/44367/4/ S1900585_es.pdf

Rowe, F. \& Bell, M. (2017). The drivers of long-distance commuting in Chile: The role of spatial distribution of economic activities and population. En J. Poot \& M. Roskruge (Eds.), Population Change and Impacts in Asia and the Pacific, New Frontiers in Regional Science. Asian Perspectives XX. https://doi.org/10.1007/978-4-431-55XXX-2_1

Sassen, S. (1991). The Global City. New York, London, Tokyo. Princeton University Press.

Sassen, S. (2007). El reposicionamiento de las ciudades y regiones urbanas en una economía global: ampliando las opciones de políticas y gobernanza. EURE, 33(100), 9-34. http://dx.doi.org/10.4067/S0250-71612007000300002

Villa, M. (1991). Introducción al análisis de la migración: apuntes de clase: notas preliminares. CELADE, Serie B, n. 91 [LC/DEM/R.I64]. https://repositorio.cepal.org/handle/11362 19271

Villa, M. \& Rodríguez-Vignoli, J. (1997). Dinámica sociodemográfica de las metrópolis latinoamericanas durante la segunda mitad del siglo xx. Notas de Población, 25(6), 17110. https://repositorio.cepal.org/handle/11362/12518?locale-attribute=en

White, M. (Ed.). (2016). International Handbook of Migration and Population Distribution. Springer. 
Williamson, J. (1965). Regional inequality and the process of national development: A description of the patterns. Economic Development and Cultural Change, 13(4), 3-47. http://dx.doi.org/10.1086/450136

Williamson, J. (1988). Migrant selectivity, urbanization, and industrial revolutions. Population and Development Review, 14(2), 287-314. https://doi.org/10.2307/1973573 